\title{
VII. HERZOGIN VON NEMOURS UND GENEVOIS
}

\author{
1. „Vous êtes tous deux si aises et contents« \\ Anna d'Este und Jacques de Savoie
}

[C]este belle, illustre et vertueuse dame [...] se remaria en secondes nopces à $\mathrm{M}$. de $\mathrm{Ne}$ mours, ce grand prince si parfaict que j'ay dict, pour s'entretenir tousjours en recherche de la perfection des honnestes marys, puisque telle estoit sa volonté de se remarier, ne faisant point comme plusieurs dames que $j$ 'ay veu vefves et convollantes, qui de leurs premiers et grands mariages s'abaissoient et descendoient fort bas avecques des petitz ${ }^{1}$.

Nicht von allen Zeitgenossen wurde Annas zweites Eheprojekt mit so viel Wohlwollen beurteilt wie von Brantôme, und er selbst erwähnt an anderer Stelle eine gegen die Witwe und ihre zweite Heirat gerichtete Schmăhschrift. Diese Vorbehalte ruhrten nicht zuletzt daher, daß die zweite oder gar dritte Ehe einer Frau von den Theoretikern des 16. Jahrhunderts entgegen der găngigen Praxis meist mit wenig Wohlwollen betrachtet wurde. So verdammt Juan Luis Vives den Wunsch einer Witwe nach Wiederheirat zwar nicht völlig, doch solle sie eine zweite Ehe niemals selbst suchen und sich, wenn überhaupt, nur ungern und gezwungen durch die Verhältnisse zur Hochzeit überreden lassen. Darüber hinaus dürfe eine weitere Ehe laut Vives nur dann eingegangen werden, wenn die Witwe, die sich eigentlich Gott zuwenden müsse, zu befürchten habe, den auf sie einstürmenden mannigfachen Versuchungen nicht widerstehen zu können ${ }^{2}$.

Obwohl der Herzog von Nemours zu den Großen des Hofes gehörte, standen zunächst vor allem die Mutter und der älteste Schwager der Braut dieser neuen Verbindung skeptisch gegenuber. Jacques de Savoie war Herzog von Nemours und von Genevois, daher sowohl an Frankreich als auch an Savoyen gebunden. Einziger Sohn von Philippe de Savoie und Charlotte d'Orléans hatte er das Licht der Welt im gleichen Jahr wie seine spätere Gemahlin erblickt. Die Frage, ob die beiden schon vor dem Anschlag auf François ein Liebesverhältnis unterhielten, ist letztlich nicht zu beantworten. Die propagandistischen Angriffe auf die Herzogin von Nemours während der Zeit der Ligue spielten jedenfalls nicht selten hierauf an. So wurde in einer mit "Le manifeste des dames de la cour

${ }^{1}$ BRANTÓME, Euvres complètes, S. 101f. Der folgende Hinweis auf die Schmähschrift: BRANTÓME, Recueil des Dames, S. 637. Vgl. Giorgio de PIAGGI, Società militare e mondo femminile nell' opera di Brantôme, Salerno 1970, S. 311.

${ }^{2}$ VIVES, Education of a Christian Woman, S. 297-326. Vgl. BeAUVALET-BOUTOUYRIE, Être Veuve, S. 229f. 
seine Mutter gerichtete Frage in den Mund gelegt: Mais, madame, puisque mon frère de Nemours et moi sommes tous d'un père et de vous, pourquoi l'aimez-vous plus que moi?, und ein anderes Pamphlet empfahl ihm die Beichtformel: 'Ecce enim in iniquitatibus conceptus sum, et in peccatis concepit me mater mear. A cause de Monsieur de Nemours, qui faisait l'amour à sa mère ${ }^{3}$. Konkrete Hinweise auf eine außereheliche Beziehung der Prinzessin finden sich nicht, wenn sich ihr Kontakt zum Herzog von Nemours nach dem Tod des ersten Gemahls auch verstärkt zu haben scheint, wie etwa der Bote vermuten läßt, welcher im Mai 1563 en dilligence sur chevaulx de poste par commandement de madite dame vers Monsieur de Nemours geeilt war. Und während der Verfolgung der Verantwortlichen für den Mord am Herzog von Guise war Jacques fast immer an Annas Seite zu sehen ${ }^{4}$.

\section{"A la fin vous serez mariés«: Schwierigkeiten der Heirat einer Witwe}

Eigentlich sollte Jacques zunächst die Schwester seiner späteren Gemahlin ehelichen. Die Idee einer Verbindung Lucrezias mit einem französischen Prinzen war schon im Januar 1550 vom Kardinal von Ferrara und den Guise, considerato l'esempio di Madama d'Omala, als wünschenswert befunden worden. In den folgenden Jahren stand auf der Liste der Kandidaten dann wiederholt der Name des Herzogs von Nemours, der sich bereits mit der Prinzessin vermählt sah, diese während eines Besuches alla franzese küßte und sich bei seinem zukünftigen Schwager über die serimounies [cérémonies] que les fames font à la fasson d'Italie beschwerte. Weniger »cérémonies« erwarteten Jacques bei Françoise de Rohan, einer Dame der Königin. Ob er der Prinzessin tatsächlich die Ehe versprochen oder sie lediglich in dem Glauben gelassen hatte, sie heiraten zu wollen - die junge Dame war davon überzeugt, die zukünftige Herzogin von Nemours zu sein. Das Kind, welches sie derweil von ihrem vermeintlichen Bräutigam erwartete, verstärkte sie nur in dieser Annahme, und als der junge Mann jede Verantwortung zurückwies, begab sich

${ }^{3}$ Die beiden Pamphleten von 1587 und 1588 in: Pierre de L'EsTolle, Journal pour le règne de Henri III (1574-1589), hg. v. Louis-Raymond LEFÈVRE, Paris 1943, S. 533, 588.

${ }^{4}$ Giulio Alvarotti an Alfonso d'Este, 29. Aug. u. 3. Dez. 1563, Paris, in: ASM, Canc. duc., Amb. Francia 38. Das Zitat: Rechnungsbuch 1563, fol. 78v.

${ }^{5}$ Ippolito d'Este an Ercole d'Este, 17. Jan. 1550, Rom, in: ASM, Casa 148; Giovan Pietro Conegrani (nicht Canigiani!) an Guglielmo Gonzaga, 19. Febr. 1557, Ferrara, in: PACIFICI, Luigi d'Este, S. 103, Anm. 1; Jacques de Savoie an Alfonso d'Este, o.D. (Ende 1556Anfang 1557), in: ASM, Canc. duc., Cart. prin. est. 1457 A/21. Vgl. François de Lorraine an Ercole d'Este, 7. Okt. 1556, Paris, in: Ibid. Nr. 1568/13; Dr. Wotton an Maria Tudor und ihre Sekretäre, 12. Apr. u. 8. Nov. 1556, in: Calendar of State Papers. Foreign Series, of the Reign of Mary, 1553-1558, London 1861, ND 1967, S. 223, 274; Giulio Alvarotti an Ercole d'Este, 8. Juni 1554, Soissons, in: FonTANA, Renata di Francia, Bd. 2, S. 358-364. 
Françoise vor Gericht mit dem Ziel, die Ehe zu erzwingen. Da die Klägerin Protestantin und darüber hinaus mit der Königin von Navarra verwandt war, genoß sie die Unterstützung der reformierten Partei ${ }^{6}$.

Für Anna wurden diese Vorgänge ab dem Zeitpunkt bedeutsam, zu dem sie sich mit dem Herzog vermählen wollte, der mittlerweile jahrealte Prozeß aber immer noch nicht abgeschlossen war und das (angebliche) Eheversprechen offiziell immer noch bestand. Anfang November 1565 wurde dann vom Erzbischof von Lyon als Beauftragtem Pius' IV. ein Urteil zugunsten des Herzogs gefällt ${ }^{7}$. Einen Tag bevor Anna und Jacques ihren Heiratsvertrag unterzeichneten, wiesen auch der König und sein Rat alle Ansprüche der Dame endgültig ab. Damit die Urteile de plus grande vertu seien, bat Katharina von Medici kurz darauf ihren Gesandten in Rom, den Papst um eine definitive Entscheidung anzugehen: vous sçavez comme j'ayme madicte cousine [Anna] et que je seroys infiniment marrye, s'estant mariée de bonne foi, de la veoyr en peine ${ }^{8}$. Doch es sollte Jahre dauern, bis die Rota ihr Urteil verkündete, und erst im Januar 1580 verzichtete Françoise, abgefunden mit dem Ort und dem Herzogstitel Loudun sowie einer Rente von 50000 Livres, auf weitere Ansprüche. In der Folge begegnete sie der wirklichen Herzogin von Nemours mit distanzierter Höflichkeit'.

Sich mit der Witwe des Herzogs von Guise zu vermählen - dieses Vorhaben scheint Jacques lange mit sich herumgetragen zu haben. Als er im Sommer 1564 die ersten Avancen wagte und Anna seine Pläne offenbarte, erhielt er von seinem Boten eine zwar amüsante, aber doch enttäuschende Nachricht: $J$ 'ai montré votre lettre à votre Dame et elle en a bien fort ri. Et pense que [si]

${ }^{6}$ Antoine de Bourbon an Jeanne d'Albret, o.D. (Ende 1556-1. H. 1557, nicht 1560!), Vendôme, in: BOURBON, ALBRET, Lettres, S. 222f.; Jeanne d'Albret an Théodore de Bèze, 6. Dez. (1566), Paris, in: BÈzE, Correspondance, Bd. 7, S. 281 f. Françoise de Rohan war die Großnichte von Jeanne d'Albret, Tochter derer Cousine Isabeau, ihr unehelicher Sohn wurde in Genf erzogen.

7 Jacques de Savoie an Cosimo de' Medici, 6. Dez. 1565, Lyon, in: Hector de LA FERRIËRE, Une cause célèbre au XVI $\mathrm{XV}^{e}$ siècle. Françoise de Rohan, in: Revue des deux mondes 53 (1882) S. 649-672, hier S. 668.

${ }^{8}$ Katharina von Medici an M. de Villeparisis, 17. Mai 1566, Saint-Maur-des-Fossés, in: MÉDICIS, Lettres, Bd. 2, S. 364.

${ }^{9}$ Françoise de Rohan an Anna d'Este, 31. Jan. 1581, Loudun, in: BnF, Fr. 3346, fol. 50r. Der Brief wurde verfaßt, nachdem Anna Françoise zu einer Heirat bewegen wollte, diese aber nur geantwortet hatte, sie wolle sich nicht zweimal vermählen: Enea Renieri da Colle an Belisario Vinta, 20. Dez. 1580, in: DesjaRdINS (Hg.), Négotiations diplomatiques, Bd. 4, S. 344f., Anm. 1. Zu Annas Bemühungen um den unehelichen Sohn ihres Gemahls: Anselmo Dandino an Tolomeo Galli, 9. Febr. 1579-20. Febr. 1581, in: Correspondance Dandino, S. 323-795. Wie wenig diese Bemühungen fruchteten und wie lange sich der Streit tatsächlich hinzog, zeigt: René de Lucinge an Carlo Emanuele di Savoia, 24. Febr. 1586, Paris, in: René de LUCINGE, Lettres sur la Cour d'Henri III en 1586, hg. v. Alain DUFOUR, Genève 1966, S. 82f. Zu den Einzelheiten des Prozesses: Alphonse de RUBLE, Le Duc de Nemours et Mademoiselle de Rohan (1531-1592), Paris 1883. 
une fois je vous montre le chemin à [tous] vous autres amoureux, qu'à la fin vous serez marié[s...]. Je ne sais quand ce sera. Mais ce ne saurait être trop tôt à mon gré. Zur gleichen Zeit schrieb der Kardinal von Lothringen seiner Schwägerin, ebenfalls scherzend, da ihm die Vorstellung, Anna würde sich wieder vermählen, absurd erschien: $j$ 'oublioys vous dire qu'encoires on vous prandroit plustost que vostre fille ${ }^{10}$. Tatsächlich sträubte sich die Witwe gegen eine weitere Heirat, dem König und seiner Mutter teilte sie ihren Wunsch mit, unverheiratet zu bleiben, und dem Herzog von Nemours sagte sie ins Gesicht, daß sie als Witwe des Herzogs von Guise sterben würde, worauf Jacques wütend den Hof verlie ${ }^{11}$. Ein Jahr spatter hatte sich die Situation kaum verändert. Der Herzog von Nemours schrieb weiterhin Briefe an all diejenigen, die Anna in ihrer Entscheidung zu seinen Gunsten beeinflussen konnten, doch die Antworten blieben verhalten. Charles de Lorraine versicherte lediglich: je ne vous donneray occasion de mescontentemant, und Luigi d'Este wollte sich zuvor mit der Mutter und dem Bruder abstimmen. Letzterem versicherte Jacques, sein großßter Wunsch sei es, sein Schwager zu sein, da er jedoch wußte, daß sein Streit mit Françoise de Rohan der Grund fur die Zuruckhaltung des anderen war, fügte er hinzu: $j$ 'espère estre bientost [h]ors de mon procès - dieu sest [sait] combien il m'annuie ${ }^{12}$.

Nachdem der Erzbischof von Lyon seine Entscheidung zugunsten des Herzogs getroffen hatte, schienen alle Hindernisse aus dem Weg gerăumt. Jetzt liege es nur noch an ihr $\mathrm{zu}$ entscheiden, ob sie wieder heiraten wolle, teilte Katharina ihrer Freundin mit, denn Jacques habe seinen Prozeß gewonnen ${ }^{13}$. Die Angehörigen der Witwe zeigten sich jedoch auch weiterhin reserviert. So versicherte der Kardinal von Lothringen, nachdem der Herzog ihn über den Ausgang seines Prozesses unterrichtet hatte, er verstehe

la poine où vous estes pour le desir que vous aves d'epouser madame ma seur, ce que, Monsieur, je ne trouveray jamays estrange car Dieu l'a douée de tant de grandes et vertueuses qualités qu'elle merite autant que personne que j'ay jamais conneue. Vray est, Mon-

${ }^{10}$ Florimond Robertet an Jacques de Savoie, 10. Aug. 1564, Roussillon, in: Polzat, Princesse de Clèves, S. 55. Die Wörter und Wortteile in Klammern wurden von mir anhand BnF, Fr. 3211, fol. 8r korrigiert; Charles de Lorraine an Anna d'Este, 18. Aug. 1564, Moyen, in: LORRAINE, Lettres, S. 514.

${ }^{11}$ Francisco de Alava an Philipp II., 25. Okt. 1564, Marseille, in: Archivo documental Espanol, Bd. 6, S. 461; Renée de France an Katharina von Medici, o.D. (Apr. 1566), in: BnF, Fr. 3002, fol. 81r: Madite fille $n$ 'a pas la volunté de ce marier. Renée beruft sich hier auf ein Gespräch in Avignon im Herbst 1564.

${ }^{12}$ Charles de Lorraine an Jacques de Savoie, 6. Okt. (1565), Dieulouard, in: LORRAINE, Lettres, S. 523; Jacques de Savoie an Alfonso d'Este, o.D. (Okt.-Nov. 1565), in: ASM, Canc. duc., Cart. prin. est. 1457 A/21.

${ }^{13}$ Katharina von Medici an Anna d'Este, Ende Nov. 1565, in: MÉDICIS, Lettres, Bd. 10, S. 162. 
sieur, que je ne me suis, jusques icy, aperceu en fasson du monde qu'elle eust opinion de se marier et vous jure, Monsieur, que jamais ne $m$ 'an parla ${ }^{14}$.

Er selbst wolle daher zu seiner Schwägerin fahren und ihren Willen in dieser Angelegenheit ergrlinden, der, wie es weiter heißt, ne pourra estre que telle que je ne voudroys panser l'empescher ou contrarier en chose du monde ni aussi, Monsieur, la presser contre sa volunté de laquelle ceste resolution depand du tout. Wie an kaum einer anderen Stelle sind hier die Möglichkeiten abzulesen, die sich der Herzogin von Guise durch den Tod ihres Gemahls eröffnet hatten. Als Witwe genoß sie volle juristische Mündigkeit, konnte frei uber sich entscheiden und Verträge unabhängig von der Politik von Vătern und Bridern schließen. Charles ließ sich schließlich, sicher auch bedingt durch die großzügigen Angebote des Herzogs zugunsten der Kinder aus Annas erster Ehe, umstimmen und bemühte sich in der Folge gar, seine Schwägerin von dieser Verbindung zu überzeugen ${ }^{15}$.

Anders Renée de France, die bis zum Schluß gegen eine Heirat ihrer Tochter mit dem Herzog von Nemours war. Dessen Prozeß sei zwar vom Lyoner Erzbischof entschieden, aber: la preuve en est difficile. Sie wolle verhindern, ließ Renée die Königin wissen, daß Anna der Name einer Ehebrecherin angehăngt whirde:

Je ne puis ny doibs taire, tandis que je congnoys que votredite majesté y peut remedier et pourvoir, estant bien certaine que madite fille n'a pas la volunté de ce marier comme elle a de vous obeyr, [...] je voy que toutes les aydes et faveurs du monde ne liny leveront jamais le nom qui est trop à hayr et fuir, et voy qu'on luy donnera si le mariage se faict ${ }^{16}$.

Doch Katharina, die das Eheprojekt von Anfang an unterstiutzt hatte, versprach, daß der König, welcher außerdem eine große Summe zu Annas Mitgift beisteuern wollte, und sie selbst solche Sorgfalt auf den Heiratsvertrag verwenden würden, daß sie zufrieden sein könne. Drei Zeilen weiter erinnert die Florentinerin an quelques ministres que vous avez auprès de vous, was sicher als Versuch angesehen werden kann, mittels eines Hinweises auf die gegenuber Renées religiöser Praktiken waltende Toleranz ihre Zustimmung zu erzwingen ${ }^{17}$.

Für Anna stand fest, keine neue Ehe einzugehen, bevor nicht der Prozeß gegen die Mörder ihres Gemahls beendet sei. Als dann im Januar 1566 das Urteil gesprochen war, verlor die Prinzessin keine Minute, die Familien um ihr

${ }^{14}$ Charles de Lorraine an Jacques de Savoie, 2. Dez. (nicht Nov.!) 1565, Reims, in: LORRAINE, Lettres, S. 524. Das folgende Zitat: Ibid.

${ }^{15}$ Ippolito d'Este an Anna d'Este, 20. März 1566, Rom, in: MÜNCH (Hg.), Denkwürdigkeiten, S. $122 f$.

${ }^{16}$ Renée de France an Katharina von Medici, o.D. (Apr. 1566), in: BnF, Fr. 3002, fol. 81 r.

11 Katharina von Medici an Renée de France, April 1566, in: MÉDICIS, Lettres, Bd. 10, S. 168. 
Wohlwollen zu bitten. Sowohl die Este als auch die Lothringer zeigten sich mit dem Eheprojekt einverstanden. Während Maria Stuart die grandes offres lobte, die der Herzog ihren Cousins gemacht hatte, und sie Anna als eine der glücklichsten Frauen der Welt bezeichnete, stimmten andere dem Heiratswunsch nicht zuletzt in Anbetracht der Person des Bräutigams zu: Wenn sie Lust habe, sich wieder zu vermählen, könne sie keine ehrenvollere Partie finden, da Jacques ein Fürst sei, der von aller Welt geschätzt würde ${ }^{18}$. Seit 1560 schützte das Édit des secondes noces im Falle der Wiedervermählung einer Witwe die Rechte ihrer Kinder aus erster Ehe, doch Annas Zugeständnisse gingen weit über das Verordnete hinaus. Ihre Söhne Henri, Charles, Louis, François und Maximilien erhielten eine großzügige Schenkung und wurden selbst im neuen Heiratsvertrag auf außergewöhnliche Weise bedacht. Die Tochter Catherine ging so gut wie leer aus. Am 29. April 1566 überschrieb die Herzogin ihren Söhnen Land und Herrschaft von Saumur, Provins und Dourdan sowie die Hälfte der Grafschaft Nanteuil, außerdem ihren gesamten Schmuck. Neben einem lebenslangen Nutzrecht hatte sich Anna einige kleinere Privilegien vorbehalten, darunter die Möglichkeit, ihre Tochter mit einer Mitgift auszustatten ${ }^{19}$.

Am selben Tag unterzeichnete das Brautpaar, in Anwesenheit der königlichen Familie sowie mehrerer Kardinäle, Fürstinnen und Fürsten, ihren Ehevertrag. Die zukünftige Herzogin von Nemours erhielt eine Mitgift von 100000 Livres, die in voller Höhe vom König übernommen wurde; der Herzog von Savoyen garantierte ihr douaire und machte darüber hinaus dem Bräutigam ein großzügiges Geschenk ${ }^{20}$. Als Wohnung für den Fall, daß ihr Gemahl vor ihr sterben sollte, wurde der Prinzessin das Schloß von Annecy oder ein anderes Haus ihrer Wahl zugewiesen. Wie groß der Wunsch des Herzogs von Nemours war, Anna zu seiner Gemahlin zu machen, wird an den Zugeständnissen deutlich, die er ihren Sprößlingen aus erster Ehe zu machen bereit war. Neben den üblichen Vereinbarungen enthielt der Ehevertrag das Versprechen des Herzogs, nicht nur meubles, bagues et joyaulx, sondern seinen französischen Grundbesitz und alles, was ihm im Königreich gehöre, die gesamte Mitgift seiner Gattin sowie alle Käufe, die das Paar gemeinsam oder jeder für sich während der Zeit ihrer Ehe tätigen würde, den Erben Annas zu vermachen für den Fall, daß ihm selbst aus dieser Verbindung keine Nachkommen gebo-

${ }^{18}$ Maria Stuart an Anna d'Este, Mai 1566, Edinburgh, in: STUART, Lettres, Bd. 1, S. 354; Nicolas de Lorraine an dies., 4. Apr. (1566), Pont-Saint-Vincent, in: BnF, Fr. 3200, fol. 77r. Vgl. die Briefe von Luigi Pico della Mirandola, Ippolito d'Este und Charles, Herzog von Lothringen, 15. Febr., 20. März, Anfang Apr. 1566, in: BnF, Fr. 3211, fol. 66r, 73r, Fr. 3391, fol. 80r.

${ }^{19}$ Schenkung von Anna d'Este an ihre männlichen Kinder, 29. Apr. 1566, in: AST (Cast.), Int., PGN, cat. 5, m. 6, n. 4. Vgl. BRIÈLE (Hg.), Collection de documents, S. 202.

${ }^{20}$ Garantie und Schenkung von Emanuele Filiberto di Savoia für Jacques de Savoie, 12. Febr. 1566, Turin, in: AST (Cast.), Int., PGN, cat. 2, m. 10, n. 1. 
ren würden. Die Braut ließ sich von ihm darüber hinaus garantieren, die durch den Tod ihres ersten Gemahls erhaltene volle Mündigkeit auch weiterhin genießen zu können: Et en ce faisant demeure ladite dame future espouze en ladministration de tous ses biens tant meubles que inmeubles presens et advenir, [...] ayant lentiere et plaine adimistration d'iceulx, et de pouvoir contracter et [ester] en jugement faire poursuittes et soy deffendre en jugement et dehors. Tatsächlich benötigte die Prinzessin für Vertragsunterzeichnungen in den folgenden Jahren nicht mehr die Vollmacht ihres Gemahls, sondern sie war auctorisée irrevocablement par son contract de mariage ${ }^{21}$.

Am folgenden Sonntag wurde in Saint-Maur-des-Fossés die Hochzeit gefeiert, et pour rentre la célébration plus solemnelle, et plus autentique, le Roi et la Reine sa mere voulurent bien y assister ${ }^{22}$. Die Bemerkung de Thous ist von Bedeutung, zeigt sie doch, daß es einer besonderen Bestätigung dieser Heirat bedurfte, da Françoise de Rohan trotz der Urteile des päpstlichen Beauftragten und des Königs an ihren Forderungen festhielt. Die Protestanten und einige "Papisten « seien dagegen, war in England zu erfahren, während im französischen Königreich satirische Schriften zirkulierten ${ }^{23}$. Die Vorgänge um die Hochzeitszeremonie sollten dann einer gewissen Komik nicht entbehren. Zur Verwirrung der Gegner und Vorbeugung unerfreulicher Zwischenfälle waren Altar und Dekorationen für die Zeremonie in einem Gebäude des ehemaligen Klosters aufgebaut, auf dessen Gelände sich das Schloß von SaintMaur befand, tatsächlich sollte die Feier aber in einem Raum des Schlosses selbst stattfinden. Obwohl der Ortswechsel dem Hof erst kurz vor der Zeremonie mitgeteilt worden war, hatte es ein gewisser Simon Petit, Pariser Jurist und Beauftragter von Françoise de Rohan geschaff, sich in den Saal zu schleichen und dort zu verbergen. Als der Kardinal von Lothringen mit der Vermählung beginnen wollte, trat Petit aus seinem Versteck hervor und rief tout hault et clair, daß er von Madame de Rohan, femme et espouse de $M$ le duc de Nemours geschickt sei, et qu'elle s'opposoit que l'on ne passast point oultre, et, quoy que ce soit, empeschoit le mariage. Die Klägerin scheint darauf gehofft $\mathrm{zu}$ haben, durch handfestes Eingreifen mehr erreichen zu können als durch Bittschriften und Prozesse ${ }^{24}$.

${ }^{21}$ Heiratsvertrag von Anna d'Este und Jacques de Savoie, 29. Apr. 1566, Monceaux, in: MüNCH (Hg.), Denkwürdigkeiten, S. 320-323. Das Wort in Klammern wurde von mir anhand AST (Cast.), Int., PGN, cat. 2, m. 10, n. 1 korrigiert. Ratifikation des Vertrags vom 11. Mai 1583 zwischen Anna und Alfonso, das mütterliche Erbe betreffend, 8. Sept. 1583, in: ASM, Casa 334.

${ }^{22}$ THOU, Histoire universelle, 1742, Bd. 3, S. 665.

${ }^{23}$ Thomas Hoby an William Cecil, 4. Mai 1566, Paris, in: Calendar Elizabeth, 1871, S. 60. Vgl. den Hinweis auf die gegen diese Heirat protestierenden lateinischen Verse in: Ibid. S. 77.

${ }^{24}$ GASSOT, Sommaire mémorial, S. 100. 
Die Hochzeitsgesellschaft ließ sich von dem Schauspiel nicht beeindrucken. König und Königin drohten, Petit hăngen zu lassen, worauf er mit gran ruido $y$ alteración antwortete. Schließlich wurde der Störenfried abgefuhrt und die Zeremonie vollzogen. Petit wurde zwar ins Gefängnis geworfen und kurz darauf wieder freigelassen, doch sollte dies nicht der letzte unerfreuliche Vorfall in Zusammenhang mit Annas zweiter Heirat sein. Rund zwei Wochen später kam es zwischen der neuen Herzogin von Nemours und der Königin von Navarra zu einem Streit, in dessen Verlauf sich die beiden Frauen palabras mayores en presencia de media $\mathrm{Co}[r t]$ e an den Kopf warfen. Während eines darauffolgenden Besuchs der Königin bei Renée de France ließ diese sie zunächst drei Reverenzen machen, bevor sie ihr Beachtung schenkte, und als Jeanne d'Albret die Freundin zur Begrußung küssen wollte, wandte Renée sich ab mit der Begründung, che mai bocca che non fusse più verdadiera che la sua non se haprosciarebbe, worauf auch hier wieder palabras vituperosas ausgetauscht wurden. Selbst noch nach der Geburt von Annas erstem Kind aus dieser Ehe bestanden die Unstimmigkeiten fort, da Jeanne kritisierte, daß Katharina von Medici den Knaben über das Taufbecken zu halten gedachte ${ }^{25}$.

\section{Der Empfang in Annecy}

In den folgenden Wochen erreichten Gratulationsschreiben und Präsente die neue Herzogin von Nemours. Das Paar wünschte nun nichts mehr, als den Hof zu verlassen und sich nach Savoyen zurückzuziehen, ma la Regina non le a voluto dare lisentia, wie es noch Ende Mai hie ${ }^{26}$. Grund fur diese Maßnahme waren neue Spannungen zwischen den Häusern Guise und Châtillon, die zuvor aus der Welt geschafft werden sollten. Erst im Juni konnten Anna und Jacques den Hof verlassen, sie waren Ende des Monats in Lyon und hielten zwei Wochen spăter mit großem Gefolge, tant de gens de chevaux arquebusies picquiers [...] et corcellets Et grand multitude de gens tant gentilshommes que aultres plusieurs gens dEglise, ihren triumphalen Einzug in Annecy, der Hauptstadt ihres Savoyer Herzogtums. Begleitet wurden die Neuvermählten von den Kardinälen von Lothringen und Guise und von Antoinette de Bourbon, außerdem von Annas Tochter Catherine, die in jenen Tagen ihren funf-

\footnotetext{
${ }^{25}$ Francisco de Alava an Philipp II., 7., 19., 25. Mai 1566, 13. Febr. 1567, Paris, in: Archivo documental Español, Bd. 8, S. 351, 359, 370, Bd. 9, S. 139; Factum zum Stand der Dinge im Prozeß Jacques de Savoie vs. Françoise de Rohan, o.D. (ca. 1570), in: BnF, Fr. 3215, fol. 71-78, hier fol. 78r; Ercole Gianelli an Alfonso d'Este, 2. Juni 1566, Paris, in: FONTANA, Renata di Francia, Bd. 3, S. 145.

${ }^{26}$ Renée de Lorraine, Ippolito und Don Francesco d'Este an Anna d'Este, 20. u. 27. Mai, 28. Okt. 1566, in: BnF, Fr. 3211, fol. 76r; MONCH (Hg.), Denkwlirdigkeiten, S. 124f.; BnF, Fr. 3234, fol. 13r. Das Zitat: Ercole Gianelli an Alfonso d'Este, 29. Mai 1566, Paris, in: FonTANA, Renata di Francia, Bd. 3, S. 140.
} 
zehnten Geburtstag begehen sollte, und einem ihrer Söhne, wahrscheinlich Charles, sowie von tota illarum familiarum clientela. Während ihres Einzuges bewegte sich die Herzogin unter einem Baldachin, Zeichen der Herrschaft und der Souveränität und daruber hinaus ein Privileg, das ihre Nachfolgerin sich einst würde erstreiten müssen ${ }^{27}$.

Als die Gesellschaft am Tor angelangt war, präsentierte sich ihr ein Vertreter der Stadt, welcher der Füstin im Namen ihres neuen Herzogtums eine Rede hielt, in der er versprach, ihr stets treu dienen und gehorchen zu wollen. Im Gegenzug solle Anna des Landes Zuflucht sein, son bien et son soulagement, et le remettre en temps passé si plain de doulceur et de grace. Die größte Bitte des Stadtrats bestand jedoch darin, ihm seine Privilegien und juristische Selbstverwaltung zu bestätigen. Darauf erschien eine junge Frau vor der Prinzessin und präsentierte ihr die Schlüssel der Porte du Bœuf. Hier endeten die Vorstellungen der Stadt, obwohl ursprünglich weitaus mehr geplant gewesen war. So war etwa die Anfertigung eines großen Bildes vorgesehen, auf dem das Urteil des Paris dargestellt sein sollte, où sera le pourtraict ou effigie de Madame au plus près du naturel que fere se pourra, oder die Abbildung des Flusses Po, wie er in den Alpen entspringt und durch die Lombardei bis nach Ferrara fließt, et de là ung bras du Po se regorgera contre la surce et se jettera, par le milieu des Alpes, dans ung lac pour figure du mariage de leurs excellences $^{28}$. Doch fur allegorische Eskapaden fehlte es an finanziellen Mitteln. Und so hatte Anna wenige Wochen nach ihrem Einzug ihren ersten Akt als Beschützerin und Fürsprecherin der Bewohner Annecys zu vollbringen. Denn der Stadtrat präsentierte seiner neuen Herrin ein Gesuch im Namen der Gemeinde, tendant aux fins qu'il plaict à leurs exellences avoyr en recommandation leurs pouvres subjectz bourgoys. Und tatsächlich: Annecy wurde vom Herzog fur den Zeitraum von einem Jahr von der Salzsteuer befreit ${ }^{29}$.

Anna hatte sich ihren Untertanen mit einer Mischung aus Macht und Milde vorgestellt. Mit einer großen Gruppe Geharnischter und Bewaffneter war sie in Annecy eingezogen, begleitet von Vertretern einer der mächtigsten Famili-

${ }^{27}$ Auszug aus dem Aktenregister des Annecyer Notars Pernod, 17. Juli 1566, in: Claude-A. Ducis, Entrée de Jacques de Savoie et d'Anne d'Este à Annecy, in: Revue savoisienne 24 (1883) S. 16f., Zitat S. 17; Théodore de Bèze an Johannes Haller, 16. Juli 1566, Genf, in: BĖZE, Correspondance, Bd. 7, S. 182. Zum Baldachin: Mémoire historique sur la ville d'Annecy et sur les droits des ducs de Genevois et de Nemours, o.D. (Mitte 17. Jh.), in: BnF, Fr. 32887, fol. 31vf. Annas ältester Sohn Henri befand sich zu diesem Zeitpunkt auf dem Weg zum Kaiser.

${ }^{28}$ Register der Beschlüsse des Stadtrats von Annecy, Juli 1566, in: AMA, BB 11, vol. 12, fol. 25v, 27r, 28r. Eines der Bilder, mit denen das Schloßtor dekoriert war, wurde von Anna über zwanzig Jahre lang aufbewahrt: Inventayre des meubles et armes consistantz au chasteau de la presente ville d'Annessy pour rendre à Messieurs de la chambre des comptes de Genevois, 18. Aug. 1588, in: ADA, SA 96, 18, Nr. 6, fol. 13v.

${ }^{29}$ Register der Beschlüsse des Stadtrats von Annecy, 4. Aug. u. 20. Sept. 1566, in: AMA, BB 11, vol. 12, fol. 41v, 53 f. 
en Frankreichs. Doch kurz darauf hatte sie von ihrem Gemahl und dessen Lehnsherrn, dem Herzog von Savoyen, eine Verringerung der auf den Schultern ihrer Schutzbefohlenen lastenden Abgaben erlangt. Rund zwei Jahre nach ihrer Vermählung erklärte Jacques seine Gemahlin für die Organisation des Herzogtums betreffende Fragen zu seiner procuratrice générale et spéciale mit plain pouvoyr, auctorité et puissance; aber Anna ließ die Geschäfte lieber von ihren Verwaltern regeln und blieb selbst in Frankreich ${ }^{30}$. Tatsächlich sollte sich die Prinzessin in den folgenden Jahrzehnten selten, und die letzten zwanzig Jahre ihres Lebens überhaupt nicht mehr im Genevois aufhalten, anders als ihr Gemahl, der, da er sich Hoffnungen auf eine Nachfolge in Savoyen machte, mehrere Monate des Jahres in Annecy oder auf einem seiner Schlösser in Piemont verbrachte und von dort aus die Abwesenheit seiner Frau beklagte: Il y'a longtemps que de jour à autre j'attens ma femme jcy, mais elle ne scayt venir ${ }^{31}$.

Ihre Flitterwochen verbrachten die Neuvermählten in Annecy. Es waren dies die ersten Tage und Nächte, die Anna und Jacques allein und ungestört verbringen konnten, und sie waren, wie es scheint, sehr verliebt. Vous êtes tous deux si aises et contents en Savoie que je n'ose vous faire plus longue lettre pour ne vous empêcher de la lire, schrieb ihnen ein Freund des Herzogs, und die Königinmutter wurde ungeduldig, als das Paar Anfang September immer noch nicht an den Hof zurückgekehrt war: je suys eun peu marrye contre vous de set qu'yl m'a dist que aystes grosse, de peur que j'é que ne revenyés si tot que m'avés promys; cet que vous prie fayre, et qu'i[l] n'y ayst ny enfant, ny mary qui vous enn an guarde ${ }^{32}$. Anna war tatsächlich schwanger, aber natürlich hielten weder ihr Gemahl noch ihr dicker Bauch sie davon ab, an den Hof zurückzukehren. Zu Weihnachten zog sich die werdende Mutter auf ihr Schloß in Nanteuil zurück, wo sie fast auf den Tag genau neun Monate nach der Hochzeitsnacht ihrem achten Kind, einem Sohn, der den Namen Charles-Emmanuel erhielt, das Leben schenkte ${ }^{33}$.

${ }^{30}$ Vollmacht von Jacques de Savoie für Anna d'Este, die Verwaltung von Genevoys, Foucigny und Beaufort betreffend, 5. Nov. 1568, in: ADA, SA 18699, fol. 1r. Auch als in den 1570ern um die Besetzung des Priorats von Peillonnex im Genevois gestritten wurde, schrieb Anna ihre Briefe aus Montargis: A. GAVARD, La duchesse Anne d'Este et la commende du prieuré de Peillonnex, avec trois documents inédits, 1577, in: Revue savoisienne 67 (1926) S. 27-36.

${ }^{31}$ Jacques de Savoie an Luigi d'Este, 1. Jan. 1584, Cassine Châtelier, in: ASM, Canc. duc., Cart. prin. est. $1457 \mathrm{~A} / 21$.

${ }^{32}$ Florimond Robertet an Jacques de Savoie, 29. Juli 1566, Paris, in: PolZAT, Princesse de Clèves, S. 72. Das Zitat wurde von mir anhand BnF, Fr. 3211, fol. 104r leicht korrigiert. Katharina von Medici an Anna d'Este, Sept. 1566, in: MÉDICIS, Lettres, Bd. 10, S. 179.

${ }^{33}$ Notiz über die Geburt von Charles-Emmanuel de Savoie am 7. Febr. 1567 zwischen sechs und sieben Uhr morgens im Register der Beschlüsse des Stadtrats von Annecy, in: AMA, BB 11, vol. 12, fol. 29r; Lodovico Gonzaga an Jacques de Savoie, 19. Febr. 1567, in: BnF, Fr. 3218, fol. 23r; Jacques de Savoie an Renée de France, 25. Febr. 1567, in: Ibid. fol. 27r. 


\section{Das Problem der doppelten familiären Bindung}

Die Sorge für ihren älteren Nachwuchs aus erster Ehe scheint die Prinzessin fortan dem Schwager überlassen zu haben, sicher auch angesichts der Tatsache, daß sie im Rahmen ihrer neuen Heiratsverbindung auf die Vormundschaft verzichtet hatte. Der Kardinal bemühte sich in den folgenden Jahren vor allem, die hohen Schulden seiner Neffen in den Griff zu bekommen, die, nicht anders als bei anderen Angehörigen des französischen Adels, im Laufe der Jahre enorme Ausmaße angenommen hatten. So mußten etwa die Nevers Mitte der 1560 er Jahre ihren Schmuck verstecken, um ihn vor der Pfändung zu bewahren, und den König um Hilfe gegen den Bankrott der Familie angehen ${ }^{34}$. Die Situation der Guise stellte folglich keine Ausnahme dar. Vous sçavez bien, Madame, que voz enfans sont acablés de debtes, et qu'il[s] n'ont aide de personne qui leur veulle rien donner ni payer pour eus, schrieb Charles seiner Schwägerin und fügte hinzu: Je sçay bien, Madame, qu'estans voz enfans je me ferois honte de les vous recommander. Doch er hatte verstanden, daß Anna sich nicht mehr für die mittlerweile erwachsenen und bald mündigen Kinder ihres ersten Gemahls zuständig fuhlte, und daß er selbst nun die Vaterrolle übernehmen mußte: Je suis vray bon oncle et comme [un] aultre pere de vos enfans ${ }^{35}$.

In den folgenden Jahren hatte die Prinzessin kaum noch ein Auge auf die Geschäfte ihrer Kinder, so daß deren Situation nach dem Tod des Kardinals immer aussichtsloser wurde. Charles war Ende Dezember 1574 gestorben, und die diesbezüglichen Briefe machen deutlich, welchen Stellenwert er bei Nichte und Neffen eingenommen hatte ${ }^{36}$. Die über achtzigjährige Antoinette de Bourbon war es, die nun die Rolle des »guten Onkels« übernehmen mußte. Die finanzielle Misere ihrer Enkel schilderte sie Anna in der Hoffnung, tatkrăftige Unterstützung bei der Rettung des Hauses vor dem Ruin zu erhalten: Henri sei gezwungen, jeden Monat ein Stück Land zu verkaufen, daher schreibe sie ihr, pour vous prier estre moien et tenir la main qu'il soit donné ordre. Von der Gemahlin ihres Sohnes wurde Anna versichert, sie sei ihre einzige Hoffnung, und ihre Schwägerin erinnerte sie daran, daß von ihr als Mutter alles abhinge: Or, il n'y a plus de bon oncle, tout depant de vous, vous estes la mère, je croy

Zur Taufe: Ippolito Galvagno an Guglielmo Gonzaga, 24. Jan. 1567, Paris, in: ASMan, AG 654, fol. 115r; Avviso aus Frankreich, o.D. (Febr. 1567), in: ASF, MP 4850, fol. 319r.

${ }^{34}$ CROUZET, Crise de l'aristocratie, S. 29.

${ }^{35}$ Charles de Lorraine an Anna d'Este, 11. Nov. (1568), 4. März (1569), Orléans, Metz, in: LORRAINE, Lettres, S. 581 u. BnF, Fr. 3232, fol. 12v, 14 r.

${ }^{36}$ Louis de Bourbon-Montpensier an Anna d'Este, 10. Jan. (1575), in: BnF, Fr. 3200, fol. 3r; Katharina von Medici an dies., 23. Dez. 1574, Avignon, in: MÉDICIS, Lettres, Bd. 5, S. 109;

Dies. an Jacques de Savoie, 23. Jan. 1575, Lyon, in: Ibid. S. $111 \mathrm{f}$. 
$q u$ 'il ne vous désobéira point ${ }^{37}$. So schritt die Herzogin von Nemours, ausgestattet mit einer Vollmacht ihrer Söhne, im September 1578 zur Tat und verkaufte die Grafschaft Nanteuil, welche sie einst gemeinsam mit François erworben und deren Schloß zu ihren Lieblingsresidenzen gehört hatte ${ }^{38}$.

Ähnlich verhielt es sich bei der Heirat ihrer Tochter Catherine. Der Kardinal von Lothringen übernahm die Heiratsverhandlungen für seine Nichte, kümmerte sich um deren Mitgift und unterrichtete Anna in ausführlichen Briefen uber den Fortgang des Vorhabens ${ }^{39}$. Beim Bräutigam handelte es sich um denselben Louis de Bourbon, Herzog von Montpensier, den Charles bereits eine Woche nach der Geburt seiner Nichte als mögliche Heiratspartie ins Gespräch gebracht hatte. Der Herzog war weit über fünfzig Jahre alt und mithin ălter als Catherines Vater, wäre dieser noch am Leben, während die Braut noch keine zwanzig zählte, und so dachten viele: nella età vi havrei desiderato maggior proportione ${ }^{40}$. Der Spott tiber notre amoureux, wie der Herzog von Montpensier in der Korrespondenz seiner zukünftigen Schwäger genannt wurde, ließ daher nicht lange auf sich warten. So schrieb Annas Sohn Charles dem Herzog von Nemours, der seiner Gicht wegen wieder einmal nicht am Hofleben teilhaben konnte: il n'y a rien si amoureux que Monsieur de Mompensier, et ne fait que danser tous les jours avec ma seur, und der Kardinal von Guise meinte hinsichtlich des Treffens, bei dem offiziell um die Hand seiner Nichte angehalten werden sollte, daß sie alle sicher viel über den Verliebten zu lachen haben würden, et croyes que Madame votre femme et la petite font bien les ampechées et sont tout ce matin pour tiffer la mariée. Quant à moy je feré tout mon pouvoir pour oir de quel façon il presantera son service, et ne puis pançer qu'il ne le presante de quelque hault stille ${ }^{41}$.

Ob Anna dieser Verbindung zugestimmt hätte oder tatsächlich hat, ist unklar. Sie steuerte 20000 Livres zu Catherines Mitgift bei, außerdem ein Kleid, mehrere Unterröcke und eine große Anzahl von Schmuckstücken und Edel-

${ }^{37}$ Antoinette de Bourbon an Anna d'Este, 1. Juli 1577, Joinville, in: BnF, Fr. 3338, fol. 86r; Catherine de Clèves an dies., o.D. (Mitte Sept. 1577), Joinville, in: BnF, Fr. 3200, fol. 70v; Renée de Lorraine an dies., 23. Aug. (1577), Joinville, in: BnF, Fr. 3233, fol. 13r. Vgl. den Brief von Antoinette de Bourbon, 22. Aug. (1577), Joinville, in: PoIZAT, Princesse de Cleves, S. 146: si cela ne vient de vous, je crains bien qu'il n'y est jamais ordre.

${ }^{38}$ Gaspard de Schomberg kaufte Nanteuil für 380000 l.t.: Antoinette de Bourbon an Anna d'Este, 15. u. 17. Sept., 2. Okt. 1577, in: BnF, Fr. 3338, fol. 104f., 108r; Jacqueline BouCHER, Art. "Schomberg, Gaspard de«, in: JOUANNA u.a., Histoire et Dictionnaire, S. 1291.

${ }^{39}$ Charles de Lorraine an Anna d'Este, 19. (Aug. 1569), Amboise, in: LORRAINE, Lettres, S. 600f.; DERS., Pour Mademoyselle de Guyse son dot, 5. Sept. (1569), in: BnF, Fr. 3232, fol. 113f., teilw. publ. in: René de BoullLE, Histoire des ducs de Guise, Bd. 2, Paris 1849, S. $589 f$.

${ }^{40}$ Ippolito d'Este an Anna d'Este, 10. Apr. 1570, Rom, in: BnF, Fr. 3229, fol. 32r.

${ }^{41}$ Charles de Lorraine, Marquis von Mayenne, an Jacques de Savoie, 29. Jan. (1570), Angers, in: BnF, Fr. 3390, fol. 7r; Louis de Lorraine an dens., 20. Dez. (1569), Bourgueil, in: BnF, Fr. 3231, fol. 32r. 
steinen, darunter: Une coiffe de rubys et de perles où y a trente roses émaillées de blanc, et en chascune rose un ruby et trois cents soixante perles caboches $^{42}$. Vielleicht gehörte zu der Hochzeitsausstattung, welche die zukünftige Herzogin von Montpensier von ihrer Mutter erhielt, auch die kostbare Unterwäsche, die einst für Anne de Bretagne angefertigt, dann an Renée de France vererbt und von dieser an ihre allteste Tochter weitergeschenkt worden war. Catherine sollte sich mit diesen beaux ouvrages en linge später bei der Schwester Heinrichs IV. bedanken, als diese ihr in den Wirren der Thronstreitigkeiten Asyl im Schloß von Saint-Germain-en-Laye gewahrte ${ }^{43}$. Nach ihrer Heirat nahm Catherine als Herzogin von Montpensier bei Hofe einen höheren Rang ein als ihre Mutter. Beim Festbankett am Abend nach der Krönung Elisabeths von Österreich etwa saß sie unmittelbar neben der jungen Konigin, während Anna zwar an derer rechten Seite, aber drei Stühle weiter plaziert war. Vier Jahre später, anläßlich der Heirat von Heinrich III. mit Luise von Lothringen, stellte sich die Rangfolge ähnlich dar: In nächster Nähe zum König dinierte die Herzogin von Montpensier, etwas weiter entfernt Antoinette de Bourbon und dann schließlich Anna, jedoch nicht, ohne zu protestieren ${ }^{44}$.

Wenige Monate nach der Vermählung von Annas Tochter sollte auch ihr ältester Sohn heiraten. Hatte man anfangs noch über eine Verbindung des Herzogs von Guise mit einer bayrischen Prinzessin nachgedacht, stand spätestens seit ihrer Rekonversion zum Katholizismus Catherine de Clèves, Gräfin von Eu, Tochter des Herzogs von Nevers und Witwe des Prinzen von Porcien als Braut fest ${ }^{45}$. Doch die Jahre zogen ins Land, ohne daß etwas geschah. Als im Frühsommer 1570 das Gerucht über eine Liebesbeziehung Henris mit der Schwester des Königs kursierte, man gar uber eine Heirat der beiden zu spekulieren begann, stand die völlige Kompromittierung der Guise bei Hofe zu befürchten, da natürlich weder Karl IX. noch seine Mutter, am wenigsten aber der Herzog von Anjou, den Margarete von Valois in ihren Memoiren als Intri-

${ }^{42}$ Inventaire des bagues que Madame de Nemours a donné à Madame de Montpensier à ses nopces comme il appert par l'inventaire lors faict et signé par devant notaires, 16. Sept. 1584, in: AST (Cast.), Int., PGN, cat. 5, m. 6, n. 6. Vgl. den Heiratsvertrag, 4. Febr. 1570, Angers, in: Ibid. m. 8, n. 1.

${ }^{43}$ Pierre-Victor PALMA CAYET, Chronologie novenaire, contenant l'histoire de la guerre sous le regne de Henry IV, Bd. 6, hg. v. Claude-Bernard PETITOT, Paris 1824, S. 60f., Zitat S. 61 (1595).

${ }^{44} \mathrm{~L}$ 'ordre tenu à l'entrée de très haulte, très puissante et très chrestienne princesse Madame Elizabeth d'Austriche, 29. Mărz 1571, in: GUÉRIN u.a. (Hg.), Registres des délibérations, Bd. 6, S. 311; Cérémonie observée à l'assiette du festin des fiançailles et nopces du Roy Henry troisiesme à Rheims, o.D. (15. Febr. 1575), Kopie des 17. Jh., in: BnF, Fr. 4321, fol. 38r; Valentine Dale an William Cecil, 18. Mărz 1575, Paris, in: Calendar Elizabeth, 1880 , S. 30.

${ }^{45}$ Charles de Lorraine und Anna d'Este an Alfonso d'Este und Katharina von Medici, 5. Nov. 1566-12. Mai 1567, in: LORRAINE, Lettres, S. 529-536; Ippolito Galvagno an Guglielmo Gonzaga, 3. Juli 1567, Saint-Germain-en-Laye, in: ASMan, AG 654, fol. 133v. 
ganten in dieser Angelegenheit darstellte, diese Verbindung erlaubt hätte. Den ganzen Sommer über versuchte der Kardinal von Lothringen, seinen Neffen zur Vernunft zu bringen: Si vostre filz veult croire conseil et estre saige, il ne sera nouvelle que de bonne chere, schrieb er der Mutter des jungen Mannes. Ende August schienen die Wogen der Empörung geglättet, und man hoffte, mit der Hochzeit von Henri und Catherine die Sache wieder ins Lot bringen zu können ${ }^{46}$. Anfang Oktober fand im Hôtel d'Anjou in Paris in Anwesenheit der Brüder des Königs, der Kardinäle von Bourbon und von Lothringen, des Herzogs von Nevers sowie von Renée de France, Anna d'Este und anderen großen Damen und Herren die Unterzeichnung des Ehevertrags statt, und im August des folgenden Jahres wurde den Guise ein Erbprinz geboren ${ }^{47}$.

Obwohl Annas Engagement für die Sprößlinge aus erster Ehe nach ihrer Wiedervermählung nachließ, blieb sie dennoch den Guise nahe, reiste nach Joinville, unterhielt Korrespondenzen mit den Schwägern und stand in Kontakt zu ihren Kindern. Auch mit Maria Stuart blieb die Prinzessin in Verbindung, wie zahlreiche Briefe der Königin beweisen, in denen sie ihrer tante Komplimente schickte, sie oder ihre Sprößlinge, que je tiens aussi chers comme propres frères de mes cousins de Guise, grußen ließ und der sie aus der Haft ihr Leid klagte ${ }^{48}$. Während Anna weiterhin mit dem gesamten Clan ihres ersten Gemahls verkehrte, beschränkte sich ihre Verbindung mit Jacques de Savoie auf die persönliche Beziehung zu ihrem Gatten, denn Jacques' Eltern waren schon lange tot, und seine einzige Schwester starb wenige Jahre nach der Heirat ihres Bruders. Daruber hinaus entstammte der Herzog dem Haus Savoyen, so daß seine Bindungen teilweise außerhalb Frankreichs angesiedelt waren.

Für alle Beteiligten war es selbstverständlich, daß die Sprößlinge des Herzogs von Guise Annas neuen Gemahl als zweiten Vater akzeptierten, und Henri hatte diesem schon bald nach dessen Heirat versichert, ihm ein gehor-

\footnotetext{
${ }^{46}$ VALOIS, Mémoires, S. 84f.; Charles de Lorraine an Anna d'Este, 26. Juni (1570), Anet, in: LORRAINE, Lettres, S. 613; Fabio Mirto Frangipani an Girolamo Rusticucci, 22. Aug. 1570, Paris, in: Correspondance du nonce en France Fabio Mirto Frangipani (1568-1572 et 15861587), nonce extraordinaire en $1574,1575-1576$ et 1578 , hg. v. A. Lynn MARTIN, Rom, Paris 1984, S. 77. Vgl. VIENNOT, Marguerite de Valois, S. 39-41.

${ }^{47}$ GASSOT, Sommaire mémorial, S. 92f. Der Heiratsvertrag, 1. Okt. 1570, in: AST (Cast.), Int., PGN, cat. 5, m. 8, n. 2. Vgl. Anna d'Este an Renée de France, o.D. (Ende Aug. 1571), in: PoIzAT, Princesse de Clèves, S. 106: Sie teilt die Geburt des Kindes mit und fügt hinzu: Je prie [à] Dieu, Madame, que tout ansi qu'il vous fait aïeule, [qu']il vous fasse bisaïeule. Annas zweitältester Sohn Charles, Herzog von Mayenne, heiratete im Sommer 1576 Henriette de Savoie: Ehevertrag vom 23. Juli, in: BoulLLÉ, Histoire, Bd. 3, S. 501f. Die Tochter des ăltesten Sohnes aus dieser Ehe wurde 24 Jahre später auf den Namen Anne getauft: Extraits des registres de l'église parroissiale de St.-André-des-Arcs à Paris, o.D. (18. Jh.), in: BnF, Clair. 987, S. 437 (28. Juli 1600).

${ }^{48}$ Maria Stuart an Anna d'Este, 1574-1581, in: STUART, Lettres, Bd. 4, S. 188, 261f., Bd. 5 , S. 43f., 273f., Zitat Bd. 4, S. 261.
} 
samer Sohn sein zu wollen ${ }^{49}$. Im Gegenzug wurde Jacques völlig in die Familie der Guise integriert, zu deren treusten Anhängern er schon immer gezählt hatte. So begab sich das Herzogspaar im Mai 1567 gemeinsam mit den Kardinälen von Guise und von Lothringen zur Kur nach Plombières, und wenn sich Anna und ihr Gatte in Paris aufhielten, waren sie oft im Hôtel de Guise anzutreffen $^{50}$. Daß sich der Spagat einer zweifachen familiären Bindung nicht immer reibungslos vollzog, lassen gelegentlich auftauchende Konkurrenz und Eifersucht unter den Halbgeschwistern vermuten, wie etwa anläßlich der Verteilung der Pfründe des Kardinals von Este einige Jahre später. Mme de Nemours s'estoyt un peu hastée de demander les biens du cardinal d'Este à l'advantage de ses derniers enfans, erklärte ein Beobachter die Empörung des Herzogs von Guise, während sich Anna zu versichern beeilte: Y'ai monstré par tant d'effets l'amytié que ye porte à mesdits premyers anfans que ye croys que personne n'an sauroit yamais douter, et eus ancores moyns que les autres ${ }^{\text {s1 }}$.

Nicht zuletzt können aus den Wappen der Prinzessin ihre vielfätigen Bindungen, an die Familien der Ehemänner, des Vaters und der Mutter, ersehen werden. Verheiratete Fürstinnen benutzten üblicherweise ein gespaltenes Wappen, welches die Herkunft der Frau mit der Familie des Gemahls vereinte, und tatsächlich zeigt die jeweils rechte Seite der von Anna benutzten Wappen die Löwen, Pferde und Einhörner der Lothringen-Guise beziehungsweise der Savoyen-Nemours. Für den Platz des Wappenbildes, welcher der eigenen Herkunft vorbehalten war, entschied sich die Prinzessin jedoch nicht einfach für den golden gekrönten, goldbewehrten silbernen Adler in blauem Feld, der seit dem 13. Jahrhundert Wappentier der Este war, sondern sie bevorzugte während ihrer Ehe mit dem Herzog von Guise die Symbole der Herzogswürde ihres Vaters: den schwarzen Doppeladler und die drei goldenen Lilien in blauem, rot-gold gezähntem Feld, die das Wappen der Este seit Niccolò III. zierten. Gleichzeitig benutzte Anna einen Siegelring, der nicht das Habsburger Wappentier, sondern den Adler der Este zeigte. Während ihrer Ehe mit Jacques de Savoie war der ihrer eigenen Herkunft vorbehaltene Platz des Wappens mit den Symbolen der väterlichen Familie geschmückt: dem Este-

\footnotetext{
${ }^{49}$ Henri de Lorraine an Jacques de Savoie, 13. Juli 1566, in: BnF, Fr. 3211, fol. 92r.

${ }^{50}$ Charles de Lorraine an Nicolas Psaume, 7. Mai 1567, Joinville, in: LORRAINE, Lettres, S. 535; Bouchefort an Renée de France, 25. Febr. 1568, Paris, in: MÉDICIS, Lettres, Bd. 3, S. 128; Rechnungsbuch 1569 (5. u. 8. Jan.). Vgl. Emanuele Filiberto di Savoia an Jacques de Savoie, 13. Dez. 1578, Turin, in: BnF, Fr. 3343, fol. 65r: J'ay trouvé fort bonne la desliberation que vous aves faict d'aller en France [...] pour aider aux affaires de Messieurs de Guise.

${ }^{51}$ René de Lucinge an Carlo Emanuele di Savoia, 27. Mai 1586, Paris, in: LUCINGE, Lettres 1586, S. 205; Anna d'Este an Luigi d'Este, 13. Sept. 1586, Paris, in: ASM, Canc. duc., Cart. prin. est. 1459/23. Zum Problem der doppelten familiären Bindung: POMATA, Storia particolare, S. 356.
} 
Adler und den Lilien. Ein Platz im Wappen der Herzogin war jedoch immer den drei goldenen Lilien in blauem Feld und somit ihrer Herkunft mütterlicherseits vorbehalten - eine Seltenheit unter den Wappen verheirateter Fürstinnen. Als in Frankreich lebende Tochter einer fille de France wollte Anna offensichtlich ihre königliche Abkunft nicht unerwăhnt lassen ${ }^{52}$.

2. »Vous êtes si utille et nessessaire«

Die neue Herzogswürde

\section{Schlösser in Savoyen und Frankreich}

Die in ihrem neuen Herzogtum gelegenen Wohnsitze der Prinzessin boten alle Voraussetzungen für ein standesgemäßes Leben. In Annecy gab es bequeme Möglichkeiten zur Jagd, einen fischreichen See, der das ganze Jahr uber Forellen, große Karpfen, Hechte und Barsche hervorbrachte, und ein Schloß, das nicht weniger prächtig ausgestattet war als die anderen fürstlichen Häuser in Savoyen und Piemont. Die Wände seiner Raume waren mit Leder oder Stoff bespannt und mit Bildern dekoriert, die Porträts und Frauenakte, Allegorien und Figuren der griechischen Mythologie, Landschaften und Jagdszenen darstellten, darunter auch ein tableau de drolerie soubscript Iheronimus Bosch. In Lingotto schlief Anna in einem mit Seidenfransen dekorierten Bett aus gelbschimmerndem Damast, sie besaß ein Spinett, eine große Sammlung kostbarer Kristallgefäße und einen Glasschrein, der mit Edelsteinen und kleinen goldenen Figuren geschmückt war. In Annecy vergnugte sich das Paar bei der Falkenjagd sowie beim Billard- und Krocketspiel ${ }^{53}$.

Auch im französischen Königreich, nicht weit von Nanteuil, dem Ort, den sie einst gemeinsam mit François gekauft hatte, erwarb Anna mit ihrem zwei-

52 Angelo SPAggiari, Giuseppe TrenTt, Gli stemmi Estensi ed Austro-Estensi. Profilo storico, Modena 1985, S. 73. Bei dem in BnF, DB 256, fol. 105bis abgebildeten Wappen handelt es sich um das Wappen der Este-Familie, nicht um das spezielle Wappen Annas. Vgl. auch Werner PaRAVICnl, Gruppe und Person. Repräsentation durch Wappen im spăteren Mittelalter, in: Otto Gerhard OEXLE, Andrea von HÜLSEN-ESCH (Hg.), Die Repräsentation der Gruppen. Texte - Bilder - Objekte, Göttingen 1998, S. 327-389.

${ }^{33}$ Mémoire historique sur la ville d'Annecy, fol. 45f., 128v, 132r; Post-mortem Jacques; Inventare des Schlosses von Annecy, 8. Aug. 1585, 18. Aug. 1588, 8. Febr. 1591, in: Max BRUCHET, Trois inventaires du château d'Annecy $(1393,1549,1585)$, in: Mémoires et documents publiés par la Société savoisienne d'histoire et d'archéologie 38 (1899) S. 315424, hier S. 394-423 u. ADA, SA 96, 18, Nr. 6, 7, das Bild von Bosch fol. 1r. Zu Einrichtung und Nutzung franzossischer Schlösser im 16. Jh.: Kristen B. NEUSCHEL, Nobel Households in the Sixteenth Century: Material Settings and Human Communities, in: French Historical Studies 15 (1988) S. 595-622. 
ten Gemahl ein Schloß: Verneuil-sur-Oise. 24000 Livres bezahlte Jacques im Februar 1575 dem Besitzer, welcher den Bau weitgehend unvollendet gelassen hatte; um die Fortfürung der Arbeiten kümmerte sich die Herzogin selbst. Die den Ort umgebende Landschaft und das Tal, in welches man von den Fenstern des Schlosses aus blickte, zeichneten sich durch große Schönheit aus, attendu que le vallon est accommodé de iardins, canaux, allees couuertes d'aulnes, et toutes circuies d'iceux canaux, auec vn estang entre lesdits iardins, et le bourg. Die Bauarbeiten verzögerten sich indes, und das Projekt wurde nie vollständig zu Ende gefuhrt; die Idee einer großen Terrasse, von der aus man das Tal hătte uberblicken können, blieb unverwirklicht. Nach Jacques' Tod, mit dem Verneuil in den Besitz seines Sohnes überging, gab die Prinzessin die Bauprojekte auf; fünfzehn Jahre später wurde das Schloß an Heinrich IV. verkauft ${ }^{54}$.

\section{Die Kinder aus der Ehe mit Jacques de Savoie}

In den ersten sechs Jahren ihrer Ehe mit Jacques sollte Anna drei Kindern das Leben schenken. Hinsichtlich der Rangfolge unter den Geschwistern und der Erziehungsmethoden lassen sich bei den Sprößlingen aus dieser zweiten Ehe ähnliche Tendenzen beobachten wie bei ihren Halbgeschwistern aus der Verbindung mit dem Herzog von Guise. Bei näherer Betrachtung der Quellen macht sich aber ein grundlegender Unterschied bemerkbar: Die emotionale Beziehung der Herzogin zu ihren drei jüngsten Kindern scheint sehr viel stärker gewesen zu sein, als es bei den sieben Nachkommen aus erster Ehe der Fall war. Erklären läßt sich dies mit den veränderten familiären Bindungen, in denen Anna seit der Heirat mit Jacques stand. Während ihrer ersten Ehe wurde die Erziehung der Kinder nicht als eine Angelegenheit allein der Mutter und des Vaters angesehen, sondern als eine Aufgabe für den gesamten Clan; und der zukünftige Herzog und seine jüngeren Geschwister verbrachten ihre Kindheit nicht hauptsächlich an der Seite ihrer Mutter, sondern in Joinville bei der Großmutter. Anders verhielt es sich, als Anna Herzogin von Nemours wurde, die Sorge um das Wohl der Nachkommen Jacques und ihr allein oblag und die Kinder tatsächlich sehr viel häufiger in Begleitung ihrer Mutter anzutreffen waren.

\footnotetext{
54 Jacques Androuet du CERCEAU, Les plus excellents bastiments de France, hg. v. David Thomson, Paris 1988, S. 119-139, Zitat S. 119; BABELON, Châteaux de France, S. 593598. Vgl. die Besitzbestätigung durch Heinrich III., 27. Jan. 1583, aus der hervorgeht, daß Verneuil Anna und Jacques gemeinsam gehörte, in: AST (Cast.), Int., PGN, cat. 5, m. 9, n. 6. Das Schloß wurde 1734 geschleift.
} 
In einem Brief an Renée beschreibt die Prinzessin ihre neue häusliche Situation, die von der schon bald sich bemerkbar machenden Krankheit des Gemahls gekennzeichnet war:

Son fils lui a servi de médecin [car il] a souvent allégé ses maux. Il le tient presque toujours tout nu couché auprès de lui, [et] semble que [Notre] Seigneur l'ait fait pour lui faire passer le temps [car il rit touryours]. Quant à Massimilion, il cause encore plus que jamais. [Ye les vous] souhaite tous deux une heure le jour pour vous faire passer le temps ${ }^{5}$.

Charles-Emmanuel wuchs also, anders als seine Geschwister aus Annas erster Ehe, bei den Eltern auf, manchmal in Gesellschaft seines um nur wenige Jahre älteren Halbbruders. Überhaupt war er häufig in Begleitung der Mutter anzutreffen, wie etwa im Herbst und Winter 1568, als der kaum Zweijährige gemeinsam mit der Prinzessin zuerst nach Lyon und dann über Montargis nach Joinville reiste ${ }^{56}$. Noch an einem weiteren Punkt ist abzulesen, in welchem Maße die Prinzessin nun selbst die Verantwortung für die Nachkommen trug und welche veränderten Anforderungen hinsichtlich der Sorge um die Kinder an sie gestellt wurden: Während Annas Familie in Italien aus den die Sprößlinge des Herzogs von Guise betreffenden Fragen größtenteils herausgehalten worden war, sollte der Bruder in Ferrara eine nicht unbedeutende Rolle bei der Erziehung der Söhne des Herzogs von Nemours spielen.

Charles-Emmanuel, benannt nach dem Sohn des Herzogs von Savoyen, trug bis zum Tod seines Vaters den Titel des Prinzen von Genevois. Die Quellen schildern ihn als fröhlichen Jungen, der die Herzen der Erwachsenen zu erobern wußte. Anläßlich eines seiner ersten Aufenthalte am französischen Hof lobten die anwesenden Fürsten seine gute Erziehung nicht weniger als seine Natürlichkeit. Vous y aves un grant garson de filz, schrieb der Kardinal von Guise dem Vater und hob hervor, der Knabe sei sy rieus, que beaucoup qui en jugent disent qu'il vous ressemble et toutes ses actions. Der König und sein Hof seien so zufrieden mit dem Jungen, qu'il est adoré en sette court ${ }^{37}$. Nicht anders als sein ältester Halbbruder hatte auch Charles-Emmanuel schon früh repräsentative Aufgaben zu erfullen, so etwa anläßlich der Taufe der Tochter Karls IX., bei welcher der Herzog von Nemours selbst nicht anwesend war, wohl aber sein figliuolo piccolo. Und nicht anders als Henri de Lorraine ver-

\footnotetext{
${ }^{55}$ Anna d'Este an Renée de France, o.D. (1567), in: PolZAT, Princesse de Clèves, S. 114. Die Wörter in Klammern wurden von mir anhand BnF, Fr. 3230, fol. 54r korrigiert.

${ }^{56}$ Anna d'Este an Renée de France, 8. Okt. (1568), Lyon, in: BnF, Fr. 3230, fol. 32r; Rechnungsbuch 1569.

${ }^{57}$ Louis de Lorraine an Jacques de Savoie, o.D., in: BnF, Fr. 3231, fol. 66r.
} 
sprach auch er der Mutter, brav seine Pflichten zu erfullen, de mieulx en mieulx tous les jours 58 .

Einen Teil seiner Erziehung erhielt Charles-Emmanuel am Hof des Herzogs von Savoyen, wo der junge Prinz von seinem neunten bis zu seinem fünfzehnten Lebensjahr wiederholt für längere Zeit lebte. Obwohl ihr Sohn von seinem Lehrer und einem größerem Gefolge nach Savoyen begleitet wurde, überwachte Anna aus der Ferne seine Erziehung. Nicht immer war sie über die Neuigkeiten erfreut: Er wisse aus ihrem letzten Brief, daß sie unzufrieden mit ihm sei, schrieb der Knabe der Mutter aus Turin und versprach, sich bessern zu wollen. Doch nicht nur der Briefkontakt zwischen Anna und CharlesEmmanuel war in jenen Zeiten intensiv, auch durch die Gouvernante des Prinzen von Savoyen wurden der Herzog und die Herzogin von Nemours über das Befinden ihres Sohnes unterrichtet. Für den Knaben und seine Eltern bedeutete der Aufenthalt am Hofe von Savoyen eine Ehre, und in ihren Dankesschreiben zeigte sich Anna stets geehrt durch die honneurs et faveurs sowie die bonne nourriture, welche ihr Sohn vom Herzog erhalte ${ }^{59}$.

Anders als Annas Söhne aus der Ehe mit François, die ihre Erziehung hauptsächlich in Frankreich erhalten hatten, war Charles-Emmanuel schon früh auch am Hofe von Ferrara anzutreffen. Als Dreizehnjähriger reiste er zum ersten Mal nach Italien, wo Herzog Alfonso ihn wie seinen eigenen Sohn zu empfangen versprochen hatte ${ }^{60}$. Über einen Monat hielt sich der junge Prinz in Ferrara auf, um dann in Begleitung von Luigi und Don Alfonso d'Este sowie weiterer Verwandter aus der Familie seiner Mutter nach Venedig weiterzureisen. Diese und die folgenden Reisen des späteren Herzogs von Nemours dienten dem Zweck, die Bindung der Nachkommen aus Annas zweiter Ehe zu ihrer Ferrareser Familie zu festigen. Dies geschah teilweise mittels der Porträts, die Jacques nach Italien schickte, vor allem aber über den persönlichen Kontakt, und Charles-Emmanuel sollte sich bei seinen Besuchen am Hofe des Onkels stets von seiner besten Seite zeigen ${ }^{61}$. Welchen Wert die Herzogin von Nemours auf das gute Einvernehmen zwischen ihrem Sohn und den Geschwistern in Ferrara legte, ist aus einem Brief abzulesen, in dem sie noch Jahre

${ }^{58}$ Antonio Maria Salviati an Tolomeo Galli, 2. Febr. 1573, Paris, in: Correspondance Salviati, Bd. 1, S. 399; Charles-Emmanuel de Savoie an Anna d'Este, 5. März 1574, Annecy, in: BnF, Fr. 3235, fol. 93r.

${ }^{99}$ Charles-Emmanuel de Savoie an Anna d'Este, 24. März 1577, Turin, in: BnF, Fr. 3338, fol. 58r. Als Beispiel für die Briefe der Gouvernante: Marie de Gondi an Anna d'Este, o.D. (1576-1578), in: BnF, Fr. 3397, fol. 54r. Vgl. mehrere, meist undatierte Briefe von Anna d'Este an Emanuele Filiberto und Carlo Emanuele di Savoia, in: AST (Cast.), Int., Let. div., Prin. div. 78. Laut PoIZAT, Princesse de Clèves, S. 148, war der Knabe 1577 nicht freiwillig in Savoyen, sondern als Geisel, wofür jedoch weder in den Quellen noch in der Literatur eine Bestătigung zu finden ist.

${ }^{60}$ Alfonso d'Este an Jacques de Savoie, 14. Dez. 1580, Ferrara, in: BnF, Fr. 3346, fol. 38r.

${ }^{61}$ Margherita Gonzaga, Herzogin von Ferrara, an Jacques de Savoie, 20. Juni 1581, Ferrara, in: BnF, Fr. 3346, fol. 90r: Bedankt sich fuir das Porträt von Jacques' jüngstem Sohn. 
nach dem ersten Aufenthalt des Prinzen in Italien diesen anlaßßich einer weiteren Reise in den Suden aufforderte, sich zu bemühen, d'avoyr le bonne gracce de Monsieur mon frerre et de Madame d'Urbin ma seur [...], et vous randre agreable et gratieus a tous le monde ${ }^{62}$. Tatsächlich zeigte sich die Verwandtschaft in Ferrara von dem jungen Mann beeindruckt und lobte ihn als ben compito delle doti dell' animo et del corpo und qualifichato [...] et ornato di quelli buoni et ottimi costumi che si possono desiderare in ogni principale cavaliere $^{63}$. Auch der Prinz von Genevois scheint von der Begegnung mit dem Bruder seiner Mutter und dessen Hof beeindruckt gewesen zu sein. In seinem Dankesschreiben präsentierte sich der junge Mann dem Onkel als getreuer Diener, und lange nach seiner Rückkehr nach Frankreich, als bei Hofe Gerüchte kursierten, der Herzog befände sich in Schwierigkeiten, bot der Fünzehnjährige seinen service an und zeigte sich bereit, mit einer bonne troupe nach Italien zu eilen, um Alfonso beizustehen ${ }^{64}$.

Einen Einblick in Annas inniges Verhältnis zu ihren jüngsten Kindern bietet schließlich ein Briefwechsel zwischen Renée de France und ihrem Schwiegersohn anläßlich des Todes von dessen kleiner Tochter. Marguerite war Anfang Juli 1569 in Paris zur Welt gekommen, ihren Namen hatte sie von der Herzogin von Savoyen erhalten ${ }^{65}$. Als sie drei Jahre später mit dem Vater in dessen Herzogtum Genevois reiste, erkrankte Marguerite. Anna, die Mann und Tochter wegen wichtiger Geschäfte nicht hatte begleiten können, erfuhr in Paris von der Krankheit ihres Kindes: la maladie de vostre petite fille, schrieb Renée in einem besorgten Brief dem Herzog, a rendu vostre femme au lict. Die Nachricht von dem schlechten Gesundheitszustand des Maddchens habe seine Gemahlin derart erschüttert, daß Jacques sowohl ihren Damen als auch seinen Leuten verbieten solle, weiterhin schlechte Neuigkeiten zu ubermitteln; Anna solle im Gegenteil, ob wahr oder nicht, von Marguerites Genesung erfahren ${ }^{66}$. Doch der Herzog hielt es fur klüger, seine Gattin auf das zu erwartende Ende vorzubereiten. Seine Erklärung für diesen Schritt ist aufschlußreich: laiant

${ }^{62}$ Anna d'Este an Charles-Emmanuel de Savoie, 6. Nov. (1585), Lingotto, in: BnF, Fr. 3230, fol. 36r. Lucrezia war Herzogin von Urbino. Vgl. das Empfehlungsschreiben für CharlesEmmanuel von ders. an Alfonso d'Este, 27. Okt. 1585, Lingotto, in: ASM, Canc. duc., Cart. prin. est. 1458/22.

${ }^{63}$ Leonora und Don Alfonso d'Este an Jacques de Savoie, 10. Jan. u. 14. Febr. 1581, Ferrara, in: BnF, Fr. 3346, fol. 44r, 58r.

${ }^{64}$ Charles-Emmanuel de Savoie an Alfonso d'Este, 22. Mai 1581, 1. Dez. 1582, Moncalieri, Paris, in: ASM, Canc. duc., Cart. prin. est. 1459/23.

${ }^{65}$ Charles de Lorraine an Jacques de Savoie, 1. Dez. (1568), Melun, in: LORRANE, Lettres, S. 588; Katharina von Medici an Anna d'Este, 8. Juli 1569, Orléans, in: MÉDICIS, Lettres, Bd. 3, S. 260; Jacques de Savoie an Emanuele Filiberto di Savoia, 20. Juli 1569, Paris, in: AST (Cast.), Int., Let. div., Prin. div. 78; Renée de Lorraine an Anna d'Este, 25. Juli (1569), in: BnF, Fr. 3233, fol. 15r.

66 Renée de France an Jacques de Savoie, 26. Juni 1572, Montargis, in: MONCH (Hg.), Denkwürdigkeiten, S. 199. 
veue si resolu an dautres anfans et males que je luy ay veu perdre que je croy quil ni ara point de mal quel le sache plus taut [tôt] que plus tart, teilte Jacques der Schwiegermutter mit. Die Rede ist von Antoine und Maximilien, zwei Söhnen aus Annas erster Ehe, deren Tod die Prinzessin wohl mit einem gewissen Gleichmut hingenommen hatte, obwohl es sich, wie der Herzog betonte, um männliche Kinder gehandelt habe ${ }^{67}$. Seine Gemahlin nahm sich den Tod ihrer Tochter sehr zu Herzen. Der Mutter erzählte sie von la peur que $y$ 'ai de m'afoller, und daß sie sich in den Louvre zurickgezogen habe, um den Kondolenzbesuchen aus dem Weg zu gehen, die nichts darstellten als un renouvellement de pleurs et ennuis pour moi. Renée, die weiteres Leid verhindern wollte, beruhigte den Schwiegersohn und spielte die Trauer ihrer Tochter herunter: Anna gehe es gut, und sie mache sich mehr Sorgen um ihren Gatten als um sich selbst ${ }^{68}$. Vier Monate später sollte die fast Einundvierzigjăhrige schließlich ihrem letzten Kind das Leben schenken, einem Sohn, der den Namen Henri erhielt und den Titel eines Marquis von Saint-Sorlin trug ${ }^{69}$.

\section{Das Verhältnis zum Herzog von Savoyen}

Nicht nur hinsichtlich ihrer Kinder, auch in der Funktion der 'Sekretärin' und politischen Repräsentantin ihres Gemahls scheint Anna als Herzogin von Nemours weitaus größere Handlungsmöglichkeiten gehabt zu haben als es wahrend ihrer Ehe mit François der Fall war. Hatte sie sich damals überwiegend der Organisation des Herzogtums und der maison gewidmet, so korrespondierte sie nun regelmäßig mit dem obersten Lehnsherm ihres Gemahls, dem Herzog von Savoyen. Vor allem mit der fortschreitenden Verschlechterung von Jacques' Gesundheitszustand wurde ihre Unterstultzung furr ihn unentbehrlich. Sătze wie »Monsieur [mon] mari m'ha comandé d'ecrirre sette lettre pour lui, [car] la goutte l'ha pris si forte« finden sich hăufig in ihren

${ }^{67}$ Jacques de Savoie an Renée de France, 4. Juli 1572, in: MƯNCH (Hg.), Denkwürdigkeiten, S. 201 .

${ }^{68}$ Anna d'Este an Renée de France, o.D. (Mitte Juli 1572), in: BnF, Fr. 3230, fol. 34r u. PolzAT, Princesse de Clèves, S. 106; Renée de France an Jacques de Savoie, 9.-10. Juli 1572, Montargis, in: MÜNCH (Hg.), Denkwulrdigkeiten, S. 202f. u. BnF, Fr. 3228, fol. 91 r. Marguerite starb am 24. Juni 1572 und wurde am 10. Juli in Annecy beigesetzt: Register der Beschlüsse des Stadtrats von Annecy, 7. Juli 1572, in: AMA, BB 11, vol. 13, fol. 59r; Aktenregister des Annecyer Notars Pierre Deserveta, 10. Juli 1572, in: BRUCHET, Trois inventaires, S. $198 f$.

${ }^{69}$ Giovan Battista Salvato an Guglielmo Gonzaga, 2. Nov. 1572, Paris, in: ASMan, AG 656; Jacques de Savoie an Renée de France, 20. Nov. 1572, Paris, in: BnF, Fr. 3235, fol. 23r. Zur Taufe: Ders. an Emanuele Filiberto di Savoia, 11. Nov. 1572, Paris, in: AST (Cast.), Int., Let. div., Prin. div. 78; Extraits des registres de l'église, S. 239 (13. Jan. 1573). 
Briefen $^{70}$. Mit dem Herzog verhandelte Anna über Renten und Verträge, sie unterrichtete uber militärische Vorkommnisse und beriet in Fragen der Heiratspolitik. In Zeiten politischer Spannungen zwischen Jacques und Emanuele Filiberto war sie es, die den Kontakt aufrechterhielt und sich mit dem Herzog oder seinen Abgesandten traf. Vor allem aber sollte sie für Emanuele Filiberto, später auch für dessen Sohn, ein wichtiges Bindeglied zum französischen Hof darstellen und mit seinen dortigen Agenten ein von gegenseitigem Vertrauen gekennzeichnetes Verhältnis pflegen. Anna brachte Briefe, übermittelte Botschaften und richtete Grüße aus, und nicht selten vertrat sie vor der Königinmutter und ihrem Sohn die Anliegen des Herzogs ${ }^{71}$. Daß dieser sich der unersetzlichen Dienste bewußt war, die seine cousine ihm Tag für Tag leistete, ist aus seinen Dankesschreiben zu ersehen. Ce ne m'a esté chose novelle de avoyr eu preuve de l'amitie que me portes par le temoinage que vous en aves rendu en l'ocasion que set presantée, versicherte er der Prinzessin und bezeichnete es als sein größtes Glück, daß sie sich zum rechten Zeitpunkt bei Hofe befunden habe, um seine Sache zu verteidigen, dont je vous en aurey perpetuelle obligation. Und während er Anna bat, auf ihre Gesundheit zu achten, beteuerte er Jacques gegenüber, wie sehr er sich ihr verpflichtet fuhle, pour le soin et protection qu'elle prend de mez affaires par dellà [d.h. in Frankreich] ${ }^{72}$. Nach dem Tod des Vaters war es der junge Herzog Carlo Emanuele, der von den diplomatischen Fähigkeiten seiner tante profitierte und sich erfreut darüber zeigte, avec quelle vivacité et afection il vous plait d'embrasser et fovoriser mes aferes, und noch Jahrzehnte später dankbar war, pour la particulière confiance à quoy m'ont obligé tant de diverses démonstrations de l'amitié que vous me portés et de la passion que vous avez à ce que me concerne ${ }^{73}$.

Auch Katharina von Medici nutzte Annas Beziehungen zum Herzog von Savoyen, um sich über die Vorkommnisse am Turiner Hofe zu unterrichten.

${ }^{70}$ Anna d'Este an Emanuele Filiberto di Savoia, 15. Nov. (1574), Lyon, in: AST (Cast.), Int., Let. div., Prin. div. 78.

${ }^{11} \mathrm{Vgl}$. die zahlreichen Briefe von Anna d'Este an die Herzöge von Savoyen und v.v., Okt. 1574-März 1583, v.a. die Korrespondenzen anläßlich der beiden großen Krisen 1578 und 1580, ausgelöst durch Jacques' Ansprüche auf die Nachfolge im Herzogtum, in: BnF, Fr. 3343 u. AST (Cast.), Int., Let. div., Prin. div. 78; Alessandro Fiaschi an Alfonso d'Este, 24. Aug. 1575, Paris, in: ASM, Canc. duc., Amb. Francia 61; Anna d'Este an Alessandro Fiaschi, 15. Juni u. 25. Juli 1578, Annecy, in: ASM, AF 32; Katharina von Medici an Anna d'Este, 19. (nicht 6.!) Sept. 1579, Grenoble, in: MÉDICIS, Lettres, Bd. 7, S. $118 \mathrm{f}$.

${ }^{12}$ Emanuele Filiberto di Savoia an Anna d'Este, o.D. (29. Juni 1579?), 13. Dez. 1578, in: BnF, Fr. 3200, fol. 99r, Fr. 3343, fol. 61r; Ders. an Jacques de Savoie, 29. Juni 1579, Turin, in: Ibid., fol. 100r.

${ }^{73}$ Carlo Emanuele di Savoia an Anna d'Este, 14. März 1584, 6. Okt. 1599, in: BnF, Fr. 3355, fol. 92r, Fr. 3418, fol.91r. Der Herzog schenkte Anna die Hallfte der Schulden, die der französische König dank ihrer Bemühungen den Herzögen von Savoyen zurückzuerstatten bereit war: Schenkung von Carlo Emanuele di Savoia an Anna d'Este, 22. Juli 1583, in: AST (Cast.), Int., PGN, cat. 2, m. 13, n. 11. 
Vor allem anläßlich der Heirat ihrer Enkelin Catalina Micaela, Tochter Philipps II., mit Carlo Emanuele war die Prinzessin, welche die junge Braut bei ihrer Ankunft in Nizza empfangen sollte, ihre wichtigste inoffizielle Informantin, an deren Meinung Katharina viel gelegen war. Auf eine Weise, welche als penetrant bezeichnet werden könnte, forderte sie die Freundin über Monate hin auf, sobald sie Catalina Micaela zu Gesicht bekäme, ein Porträt der jungen Frau zu schicken und sie byen au long und alla véryté zu beschreiben, et n'estre tent enbesognée à fayre l'honneur de la mayson. Eventuelle Befürchtungen hinsichtlich ihrer Diskretion zerstreute Katharina mit dem Versprechen, daß nur sie selbst und das Feuer Annas Briefe zu sehen bekommen würden ${ }^{74}$. Doch nicht nur die Königinmutter, auch der Bruder in Ferrara erhielt von der Prinzessin geheime Informationen hinsichtlich dieser Heirat, wie ein Zettel mit chiffriertem Text, der einem ihrer Briefe beigelegt war, beweist. Und tatsächlich wurde Anna, obwohl sie wegen der schweren Krankheit ihres Gemahls am Ende doch nicht nach Nizza reiste, vom Herzog von Savoyen regelmäßig über den Fortgang der Reise, das Befinden ihres Sohnes und - die qualités de l'infante ma femme unterrichtet ${ }^{75}$.

\section{Annas Stellung bei Hofe}

Nicht nur in Savoyen, auch am Hof von Frankreich genoß Anna nach ihrer Heirat mit dem Herzog von Nemours ein Ansehen wie eh und je. Sie unterhielt freundschaftliche Beziehungen zu den regierenden Königinnen Elisabeth von Österreich und Luise von Lothringen, wie aus deren sehr warmen und persönlich gehaltenen Briefen zu ersehen ist, und zu den Töchtern der Königinmutter, Claude de Valois und Margarete von Valois ${ }^{76}$. Katharina von Medici wollte die Freundin stets um sich haben, sie versicherte ihr: vous savés come je vous ayme, wünschte, immer librement $\mathrm{mit}$ ihr $\mathrm{zu}$ sprechen und vertraute sich

\footnotetext{
${ }^{74}$ Katharina von Medici an Anna d'Este, 1. Sept. 1584, Febr. u. Juni 1585, in: MÉDICIS, Lettres, Bd. 8, S. 214f., 238f., 309. Ein Blick in die offizielle Korrespondenz des französischen Botschafters in Spanien, der vor allem politische Einzelheiten ubermittelt, sich aber kaum über Aussehen und Wesen der Braut äußert, erklärt Katharinas Interesse, vgl. die Briefe Mărz-Mai 1585, in: Dépêches diplomatiques de M. de LONGLÉE, résident de France en Espagne (1582-1590), hg. v. Albert MOUSSET, Paris 1912.

${ }^{75}$ Carlo Emanuele di Savoia an Anna d'Este, 14. Mărz 1585, Zaragoza, in: BnF, Fr. 3367, fol. 13r. Der chiffrierte Zettel in: Anna d'Este an Alfonso d'Este, 4. Febr. 1585, Cassine Châtelier, in: ASM, Canc. duc., Cart. prin. est. 1458/22. Anna ist nicht, wie BRANTOME, Recueil des Dames, S. 609 behauptet, aus Anlaß dieser Hochzeit selbst nach Spanien gereist. ${ }^{76}$ Alle Briefe von Elisabeth von Österreich und Luise von Lothringen an Anna d'Este, in: BnF, Fr. 3238, fol. 1-11, 22-58, sind von eigener Hand der Königinnen; Briefe von Claude de Valois und Margarete von Valois an dies., in: R. de MAGNIENVILLE, Claude de France, duchesse de Lorraine (1547-1575), Paris, Lille 1894, S. 167f., 171f. u. Marguerite de VALOIS, Correspondance, 1569-1614, hg. v. Éliane VIENNOT, Paris 1998, S. 259, $319 f$.
} 
ihr auch in den größten Bedrängnissen an: je vous mende toutes mes joyes et mes anuys, et cet que je désire. Annas Anwesenheit bedeutete für die Königinmutter grent plésir, wie sie die Menschen in ihrer Umgebung wissen ließ, und wenn die Prinzessin nicht an den Hof kommen konnte, war sie es, die sich zu ihr begab ${ }^{77}$. Der englische Botschafter beobachtete die Geheimnistuerei der beiden Damen mit Mißtrauen, und auch sein Kollege aus Savoyen bemerkte wiederholt, daß Anna sehr vertraut mit der Herrscherin sei und alles, was jene erfuhre, dieser sofort zugetragen würde: Die Königinmutter, so berichtet er, avec la duchesse de Nemours et Mme de Montpensier, sont en continuelz conseilz et particuliers, avec mine triste ${ }^{78}$.

Die Dankesschreiben derjenigen, denen Anna bei der Unterbringung von Klientinnen oder deren Söhnen im Hofstaat der Königinnen behilflich war, und der Hinweis in einem nach ihrem Tod verfaßten Lobgedicht, in dem erwăhnt wird, es gäbe niemanden,

Qui n'aye ressanti des traicts de sa clemence

Les uns pour optenir quelques bienfaicts du Roy

Les autres pour avoir favorable la loy,

D'autres pour eviter un merité supplice,

Autres pour emporter quelque bon benefice,

lassen weitere Ruckschlüsse auf ihre Stellung bei Hofe $\mathrm{zu}^{79}$. Selbst die Großen wandten sich an die Herzogin von Nemours mit ihren Wünschen, wie ein Brief der Marquise von Rothelin zeigt, in dem mit einem Hinweis auf die bonne amitié que la roine vous porte um Unterstützung gebeten wird. Auch der Herzog von Guise bediente sich des Einflusses seiner Mutter, um seine Interessen beim König zu fördern, etwa im Herbst 1585, als er sich um die Freilassung einer seiner Klienten bemühte und Anna bat, den Willen des Herr-

${ }^{77}$ Katharina von Medici an Anna d'Este und Jacques de Savoie, März 1567-Jan. 1585, in: MÉDICIS, Lettres, Bd. 3, S. 15, 41, 45, 300, Bd. 5, S. 138, Bd. 6, S. 331, Bd. 8, S. 234, Bd. 10, S. 227; Francisco de Alava an Fernando Álvarez de Toledo, Herzog von Alba, 5. Jan. 1568, Paris, in: Archivo documental Español, Bd. 10, S. 142f.; Charles de Lorraine an Anna d'Este, 11. Nov. (1568), Orléans, in: LORRAINE, Lettres, S. 581; Anna d'Este an Emanuele Filiberto di Savoia, 15. Nov. (1574), Lyon, in: AST (Cast.), Int., Let. div., Prin. div. 78.

${ }^{78}$ Valentine Dale an William Cecil, 19. Apr. 1575, Paris, in: Calendar Elizabeth, 1880, S. 47; René de Lucinge an Carlo Emanuele di Savoia, 2. Febr. 1586, Paris, in: LUCINGE, Lettres 1586, S. 49, das Zitat aus dem Brief vom 7. Sept. 1587, Paris, in: René de LUCINGE, Lettres de 1587. L'année des reîtres, hg. v. James J. SUPPLE, Genf 1994, S. 281.

${ }^{79}$ LA PALUD, Discour funebre, S. 28. Die Dankesschreiben: Madeleine de Savoie an Anna d'Este, 7. Mărz 1576, in: BnF, Fr. 3338, fol. 6r; Henri de Savoie an dies., o.D., in: ADA, $1 \mathrm{~J} 881$, Nr. 11962bis. Zum Folgenden: Jacqueline de Rohan an Anna d'Este, 27. Juli 1569, Blandy, in: DELABORDE, Garpard de Coligny, Bd. 3, S. 580f. Vgl. CARROLL, Noble Power, S. 127. 
schers in dieser Angelegenheit zu erkunden ${ }^{80}$. Schließlich ist aus der Beschreibung einer ganz normalen Audienz in den Gemächern der Königinmutter die Stellung abzulesen, welche die Prinzessin bei Hofe bekleidete. An jenem Tag saß Anna auf dem Bett der Herrscherin, wăhrend ihre Schwiegertochter, die Herzogin von Guise, gemeinsam mit einigen anderen Furstinnen neben ihr stand, die restlichen Damen aber in größerem Abstand plaziert waren $^{81}$.

Die Stellung der Herzogin von Nemours bei Hofe und in der Pariser Gesellschaft kann darüber hinaus an außerordentlichen Ereignissen abgelesen werden, wie etwa an der Krönungszeremonie von Elisabeth von Österreich, bei der Anna die Schleppe der Königin trug ${ }^{82}$. In den 1580 er Jahren hielt sie mehrere Kinder über das Taufbecken, darunter auch den Sohn der Herzöge von Nevers, und einige neugeborene Mädchen erhielten ihren Namen ${ }^{83}$. An Hochzeiten und Geburtstagsfeiern nahm die Prinzessin meist an herausragender Stelle teil, abends erschien sie zum Ball bei Hofe, und sie selbst gab Bankette in ihrem Haus, zu denen der französische Hochadel geladen wurde ${ }^{84}$. Der Geheimagent des Großherzogs von Toskana berichtet von einem dieser Feste an jenem Abend im Februar 1584, an dem der Herzog von Anjou nach Paris zurückkehrte und sich auf theatralische Weise mit dem König versöhnte. Wohin fuhrte Heinrich seinen Bruder, nachdem sich die beiden um den Hals gefallen waren? Zum Hôtel de Nemours, wo tre cardinali, tutti quei di casa Lorena, duca di Nevers, e la maggior parte di queste principesse beim Essen saßen - bei Anna versammelten sich die Großen des Hofes, diejenigen, denen der König wichtige Neuigkeiten sofort mitzuteilen wünschte ${ }^{85}$.

${ }^{80}$ Vgl. Xavier LE PERSON, "Practiques« et »Practiqueurs«. La vie politique à la fin du règne de Henri III (1584-1589), Genf 2002, S. 459.

${ }^{81}$ Amyas Paulett und Henry Cobham an Elisabeth I., 17. Nov. 1579, in: Calendar Elizabeth, 1904, S. 92. Vgl. auch den Hinweis auf Annas Plazierung bei einer Klerikerversammlung 1580, in: NIEL, Porträts, S. 3, Anm. 2.

${ }^{82}$ Bref et sommaire recueil de ce qui a esté faict, et de l'ordre tenüe à la joyeuse et triumphante Entrée de Charles IX de ce nom Roy de France, en sa bonne ville et cité de Paris, avec Couronnement de Elizabet d'Austriche son espouse, Paris 1572, in: Victor E. GRAHAM, William MCALlISTER JOHNSON, The Paris Entries of Charles IX and Elisabeth of Austria 1571, Toronto, Buffalo 1974, S. 91-246, hier S. 196.

${ }_{83}$ Extraits des registres de l'église, S. 282, 294, 304f. (12. Febr. 1579, 31. Mai 1580, 16. Okt. 1581); Giulio Busini an Belisario Vinta, 13. März 1581, in: DESJARDINS (Hg.), Négotiations diplomatiques, Bd. 4, S. 358.

${ }^{84}$ Henry Cobham an Francis Walsingham, 29. Nov. 1581, Paris, in: Calendar Elizabeth, 1907, S. 387; L'Ordre qui fust tenu au service quarantain que fist Madame de Joyeuse, veuve dudict sieur deffunct, en l'église et monastere des Cordeliers de Paris où elle assista le mardy xi decembre 1587, Kopie des 17. Jh., in: BnF, Fr. 4317, fol. 285-287. Zur Hochzeit von Anne de Batarnay und Bernard de La Valette, 13. Febr. 1582: Nicolas LE RoUX, La faveur du roi. Mignons et courtisans au temps des derniers Valois (vers 1547-vers 1589), Seyssel 2000, S. 483f.

${ }^{85}$ Giulio Busini an Belisario Vinta, 20. Febr. 1584, in: DESJARdins (Hg.), Négotiations diplomatiques, Bd. 4, S. 488. Vgl. Le PERSON, Practiques, S. 44. 


\section{Der Tod des Herzogs von Nemours}

Mitte Juni 1585, im zwanzigsten Jahr seiner Ehe mit Anna d'Este, starb Jacques de Savoie. Er starb an einer Krankheit, die in den Quellen meist mit dem pauschalen Begriff "Gicht « bezeichnet wird und die sich schon vor seiner Heirat bemerkbar gemacht hatte. Monsieur de Nemours est fort tourmenté de la goutte que luy faict garder le lict, berichteten Beobachter in regelmäßigen Abständen. Oft mußte der Herzog monatelang das Bett hüten, konnte weder Arme noch Beine bewegen, hatte unglaubliche Schmerzen zu erdulden, und mehr als einmal kursierte das Gerücht von seinem bevorstehenden Tod $^{86}$. Anna litt mit ihrem Gemahl, und ihr Mitgefuhl konnte sich so steigern, daß Nahestehende sich Sorgen auch um ihre Gesundheit machten. Aus einem Schreiben der Prinzessin hatte Katharina von Medici erfahren, welche Qualen jene angesichts des kranken Gatten auszustehen hatte, daß nichts sie zufriedenstellen und ihr Freude machen könne, et que aystes enn eune continuele poyne et anuy, und sie bat die Freundin, sich zu schonen, damit sie am Ende nicht selbst krank würde, car cet nous seroyt double mal $^{87}$.

Im Sommer 1584 hatte Jacques sich in die Cassine Châtelier, sein Haus in der Nähe von Turin, zurückgezogen. Anna machte sich Anfang September auf den Weg nach Savoyen und sollte dem Gemahl bis zu seinem Tod nicht mehr von der Seite weichen, während Charles-Emmanuel, welcher sich in Spanien befand, nicht rechtzeitig an das Sterbebett des Vaters zurückkehrte. Im Frühjahr des folgenden Jahres ordnete der Herzog seine Papiere und ließ seinen letzten Willen notariell beglaubigen, am Abend des 18. Juni starb er. Anna war zum zweiten Mal Witwe geworden, ihre Trauer war grenzenlos. Durch ihre Sekretäre ließ die Prinzessin, les larmes aux yeux, Briefe an die Fürsten Europas aufsetzen, in denen sie uber die treslongue et griefve maladye, die merveilleuse patience des Kranken und seine contrition exemplaire berichtete. Angehörige und Freunde lasen aus diesen Schreiben die extraime affliction der Hinterbliebenen heraus und rieten ihr, sich dem Willen Gottes zu beugen und auf ihre eigene Gesundheit zu achten ${ }^{88}$. Von kleinen und großen Höfen, Köni-

${ }^{86}$ Jacques de Savoie an Renée de France, Carlo Emanuele di Savoia, Alfonso und Luigi d'Este, Aug. 1571-März 1585, in: MüNCH (Hg.), Denkwürdigkeiten, S. 189f.; Gaudenzio ClARETTA, Vita di Maria Francesca Elisabetta di Savoia-Nemours, regina di Portogallo, Turin 1865, S. 33, Anm. 1; ASM, Canc. duc., Cart. prin. est. 1457 A/21. Das Zitat: Avis de la cour, 16. Jan. 1566, in: BnF, Fr. 20624, fol. 79r.

${ }^{87}$ Katharina von Medici an Anna d'Este, 6. Nov. 1569, Tonnay-Boutonne, in: MÉDICIS, Lettres, Bd. 3, S. 282. Vgl. Luigi d'Este an Jacques de Savoie, 22. Apr. u. 30. Juni 1578, Rom, Tivoli, in: BnF, Fr. 3343, fol. 12r, 30r.

${ }_{88}$ Anna d'Este an Philipp II., Alfonso d'Este, Heinrich III., 19.-21. Juni 1585, in: AN, 21 Mi 150; ASM, Canc. duc., Cart. prin. est. 1458/22; BnF, Fr. 15570, fol. 120r; Catherine de Lorraine und Katharina von Medici an Anna d'Este, Juli 1585, in: BnF, Fr. 3233, fol. 31 r u. MÉDICIS, Lettres, Bd. 8, S. 339, 343. 
gen und Kardinälen, den Fürstinnen und Fürsten Frankreichs, Italiens, Spaniens und Savoyens erhielt Anna Beileidsbekundungen. Philipp II. und seine Tochter, Heinrich III. und die königliche Familie, der Herzog von Lothringen und die Guise, die Verwandten in Ferrara, der Großherzog von Toskana und der Herzog von Mantua sowie der gesamte französische Adel versicherten der Prinzessin ihr Mitgefuhl und ihre Achtung vor dem Verstorbenen. Die Damen versuchten, sie mit dem Hinweis auf Jacques' jahrelanges Leiden und seine extremen Schmerzen, von denen er nun erlöst sei, zu trösten, und sie erinnerten die Trauernde an ihre Kinder, auquelz vous estes si utille et nessessaire ${ }^{89}$.

Der Körper des Verstorbenen wurde nach Annecy gebracht und im Grab seiner Familie in Notre-Dame-de-Liesse beigesetzt. Anna war in Piemont geblieben, wo sie ihre Angelegenheiten und die ihrer Kinder regeln wollte. Testamentarisch hatte Jacques sie, gemeinsam mit dem Herzog von Savoyen, zu seiner Testamentsvollstreckerin und Kuratorin der Söhne ernannt, s'asseurant que Son Altesse [der Herzog von Savoyen], cognoissant la prudence et bonne affection de ladite dame au bien, honneur et profit de ses deux enfans, luy delaissera la charge, ensemble de l'entière administration de leurs personnes et biens. Somit war Anna, bis Charles-Emmanuel und Henri ihr fünfundzwanzigstes Lebensjahr erreicht haben würden, allein für die Verwaltung der Herzogtümer und des gesamten Besitzes verantwortlich. Doch der Herzog hatte seinen Söhnen nicht nur Land und Immobilien, Titel und Einkünfte hinterlassen, sondern auch eine Menge Schulden. Von der Haftung für diese Schulden war seine Gattin explizit ausgenommen; Anna sei, so der Text des Testaments, aucunement tenue d'en rien payer ${ }^{90}$. Tatsächlich war die Prinzessin finanziell gut abgesichert. Jacques hatte die 100000 Livres bestätigt, welche sie anläßlich ihrer zweiten Heirat vom König erhalten hatte, auBerdem garantierte er ihr eine jährliche Rente von weiteren 10000 Livres und das lebenslange Nutzrecht an seinem beweglichen Besitz. Für die Schulden des Herzogs hatte somit zwar offiziell dessen ältester Sohn aufzukommen, die Situation der Witwe vereinfachte sich dadurch praktisch aber kaum, und so klagte Anna in ihren Briefen an die Brüder auch über le pitoiable estat auquel m'a laissé feu Monsieur mon mary und die grandz affaires qui me sont demeurez sur les bras ${ }^{91}$.

${ }^{89}$ Henriette de Clèves und Catherine de Clermont an Anna d'Este, o.D. (Sommer 1585), in: BnF, Fr. 3237, fol. 94r, Fr. 3397, fol. 72r. Vgl. die zahlreichen Kondolenzschreiben vom Sommer 1585, in: BnF, Fr. 3200, 3233, 3237, 3367, 3390, 3391, 3397. Eines davon, das von Monsieur de Lansac, 3. Juli 1585, ist abgedruckt in: LELOUP, Anne d'Este, S. 17.

${ }^{90}$ Testament Jacques de Savoie. Die Akzeptierung dieser Aufgabe durch Anna d'Este, 29. Juli 1585, in: Ibid. n. 8. Zur Beisetzung: Register der Beschlüsse des Stadtrats von Annecy, 4. Aug. 1585, in: AMA, BB 11, vol. 21, fol. 103v; Claude-A. DuCIS, Annecy et la maison de Genevois-Nemours, in: Revue savoisienne 14 (1873) S. 20-23, 25-28, hier S. 27.

${ }^{91}$ Anna d'Este an Alfonso und Luigi d'Este, 3.-4. Aug. 1585, Lingotto, in: ASM, Canc. duc., Cart. prin. est. 1458/22, 1459/23. 


\section{3. »Plus mélancolique que de coustume« \\ Die zweite Witwenschaft}

\section{Das Heiratsprojekt für Charles-Emmanuel}

Nach Jacques' Tod bestand eine der dringendsten Aufgaben der Witwe darin, eine passende Gemahlin für ihren Sohn zu finden, doch Heiratsverhandlungen, die ohne Unterstutzung engerer Familienangehöriger gefuhrt wurden, gestalteten sich oft schwierig. Während die Heiratsprojekte für die Kinder aus erster Ehe von den jeweiligen Allianzen des Herzogs von Guise bestimmt waren und Anna nach dessen Tod die Entscheidungen den Schwägern überlassen hatte, war sie hinsichtlich der Zukunft ihrer jüngeren Söhne auf sich selbst gestellt. Die große, nie realisierte Verbindung des Prinzen von Genevois mit der Prinzessin von Lothringen zeigt, wie wenig Einfluß sie letztlich auf den Fortgang der Verhandlungen hatte.

Noch zu Jacques' Lebzeiten hatte Katharina von Medici für CharlesEmmanuel eine Verbindung mit einer ihrer Enkelinnen, einer der Töchter von Claude de Valois und dem Herzog von Lothringen, vorgeschlagen, Pläne, wie der Herzog von Nemours seinem Schwager schrieb, ausquelles nous donames, à la verité, quelque esperance ${ }^{92}$. Doch das Projekt sollte einige Zeit ruhen, bis sich die Situation durch den Tod von Annas zweitem Gemahl grundlegend anderte. Der Prinz von Genevois war nun Herzog von Nemours, und als Mitglied des Hauses Savoyen glaubte er, und mit ihm der französische Hof, Ansprüche auf die Nachfolge in Savoyen erheben zu können. Zur Verwunderung der Fürsten, wie der schwächliche Herzog Carlo Emanuele überhaupt sein dreiundzwanzigstes Lebensjahr hatte erreichen können, gesellte sich die Überzeugung von seiner Unfahigkeit, Nachkommen zu zeugen. Heinrich III. und seine Mutter rechneten daher mit einer baldigen Vakanz des savoyischen Thrones und einer Nachfolgeregelung zugunsten des Herzogs von Nemours, um so lieber erneuerte Katharina nun ihre Angebote für eine Verbindung des Prinzen mit ihrer geliebten Enkelin Christine, der ältesten Tochter der Herzogin von Lothringen ${ }^{93}$. So erhielt Anna nach dem Tod des Gatten nicht nur Kondolenzschreiben, sondern auch Glückwïnsche zur bevorstehenden Hochzeit ihres Sohnes, ohne daß sie selbst größere Schritte in diese Richtung unter-

${ }^{92}$ Jacques de Savoie an Luigi d'Este, 16. Jan. 1581, Lingotto, in: ASM, Canc. duc., Cart. prin. est. $1457 \mathrm{~A} / 21$.

${ }^{83}$ Henry Cobham an die königlichen Sekretăre, 19. Sept. 1580, Moret, in: Calendar Elizabeth, 1904, S. 423; Edward Stafford an Francis Walsingham, 27. Nov. 1583, 5. Sept. 1584, Paris, in: Ibid. 1914, S. 240, 1916, S. 52; Katharina von Medici an Anna d'Este, Apr. 1585, in: MÉDICIS, Lettres, Bd. 8, S. 252f. In den Briefen von René de Lucinge, ab 1585 Botschafter des Herzogs von Savoyen in Frankreich, finden sich derart viele Erwăhnungen dieses Eheprojekts, daß im folgenden nur die wörtlichen Zitate belegt werden. 
nommen hätte. Wie wenig sie auf die Entwicklung vorbereitet war, zeigt ihr Brief an den Bruder in Ferrara, in dem sie um avis et conseyl bat, estant pressée, comme vous sarres, de fayre reponse [... à] leur Mayestés et Monsieur de Lorrayne, de peur de perdre sette occasion. Kurz darauf bat sie Heinrich formal um seine Zustimmung zu dieser Verbindung ${ }^{94}$.

Anfang Oktober 1585 schien das Projekt besiegelt, und Anna, die sich noch in Savoyen befand, wurde vom König und seiner Mutter aufgefordert, ihrer grandes affaires zuliebe nach Frankreich zu kommen ${ }^{95}$. Doch dann wurde die Herzogin von Savoyen schwanger, und das glanzvolle Heiratsvorhaben begann zu wanken, wieder ohne daß die Mutter des Verlobten auf die Entwicklungen hätte Einfluß nehmen können. Anstatt sich daraufhin sofort an den Hof begeben zu können, um die Verhandlungen mit Nachdruck voranzutreiben, mußte Anna ebenso wichtiger Angelegenheiten wegen in Savoyen bleiben. Zwar unterstützten die Guise diese Verbindung ihres Halbbruders, und das, obwohl ihre Cousine Maria Stuart den eigenen Sohn als Heiratskandidaten ins Spiel zu bringen gedachte, doch letztlich hatte die Herzogin niemanden, der ihre Abwesenheit vom Hofe hătte ausgleichen können ${ }^{96}$. Da aber weder der einflußreichste Vertreter noch der angesehenste Gesandte die persönliche Prăsenz beim König und seiner Mutter aufwiegen konnte, war Anna auf die Gewandtheit ihrer Briefe angewiesen. Daruber hinaus konnte sie nichts tun, als ihre Unterhandlungen mit Carlo Emanuele zu beschleunigen und die Entscheidungen der Anderen abzuwarten. Dem Bruder schrieb sie in jener Zeit: ye suis tous les yours plus pressée de leur Majestés de m'an aller an France pour effectuer le mariage de mon fils, lequel, dieu merci, est agreable à un chacun $^{97}$. Die Verbindung wurde schließlich ohne die Herzogin von Nemours beschlossen, deren Anwesenheit nur noch fur die Unterzeichnung des Vertrags und die anschließenden Feierlichkeiten erforderlich war. Doch obwohl Anna noch Mitte Dezember 1585 eine Einigung mit Carlo Emanuele hinsichtlich des douaire für die Braut erreicht und sie Ende des Monats von Catalina Micaela

94 Anna d'Este an Alfonso d'Este, 10. Sept. 1585, Lingotto, in: ASM, Canc. duc., Cart. prin. est. 1458/22; Dies. an Heinrich III., 18. Sept. 1585, Lingotto, in: P. ZEILLER, Un projet de mariage inédit pour une des filles du duc Charles III, in: Journal de la Société d'archéologie lorraine et du Musée historique lorrain 46 (1897) S. 88-92, hier S. 89f. Die Kondolenzschreiben: Catherine und Charles de Lorraine an Anna d'Este, 1. u. 3. Aug. (1585), Montereau, in: BnF, Fr. 3233, fol. 21 v, Fr. 3390, fol. 45v.

${ }^{95}$ René de Lucinge an Carlo Emanuele di Savoia, 3. Okt. 1585, in: René de LUCINGE, sieur des Allymes, Lettres sur les débuts de la Ligue (1585), hg. v. Alain DUFOUR, Genf 1964, S. 202. Vgl. Heinrich III. und Luise von Lothringen an Anna d'Este, 4.-5. Okt. 1585, in: MÓNCH (Hg.), Denkwilirdigkeiten, S. 257 u. BnF, Fr. 3238, fol. 58r.

${ }^{96}$ Maria Stuart an Mons. de Chateauneuf, 24. Marz 1586, Chartley, in: STUART, Lettres, Bd. 6, S. 265.

${ }^{97}$ Anna d'Este an Alfonso d'Este, 5. Dez. 1585, Lingotto, in: ASM, Canc. duc., Cart. prin. est. 1458/22. 
Urlaub für ihren jüngsten Sohn erbeten hatte, damit er an diesem Ereignis teilhaben könne, sollte die Hochzeit am Ende nie gefeiert werden ${ }^{98}$.

Die Briefe des Savoyer Botschafters, der seinen Herrn in regelmäßigen Abständen uber den Fortgang der Verhandlungen informierte, zeigen, in welchem Maße sich der König und seine Mutter, die zur gleichen Zeit bei Maria Stuart die Hoffnungen auf eine Heirat des schottischen Königs nährte, der Herzogin von Nemours für ihre politischen Ziele bedienten und wie wenig Einfluß diese selbst auf die Ereignisse hatte. Nicht wenig Erstaunen muß die Lektüre dieser Schreiben hervorrufen, in denen Anna, deren politisches Handeln sonst von großer Einsicht geprägt ist, als eine den Tatsachen blind gegenüberstehende Frau erscheint, die allen Offensichtlichkeiten zum Trotz an einem einmal gefaßten Plan festhielt. Schon allein der Umstand, daß der König die Heirat ihres Sohnes wieder und wieder verschob, erst auf Ostern, dann Pfingsten und Allerheiligen, schließlich bis nach der Fastenzeit 1587, hätte sie stutzig machen müssen. Während für die meisten der bei Hofe Verkehrenden klar war: le Roy se mocque, meinten andere gar, daß die Verbindung nie zustande kommen würde ${ }^{99}$. Das größte Problem stellte die Animosität zwischen Heinrich III. und Henri de Lorraine dar, wobei sich zu der persönlichen Feindschaft nicht nur die Spannungen zwischen den mignons des Königs und den Leuten des Herzogs gesellte, sondern vor allem Henris Weigerung, ein Friedensabkommen zu unterzeichnen. Heinrich und seine Mutter wußten, daß der König von Navarra diesbezügliche Verhandlungen niemals akzeptieren würde, tăte der Herzog von Guise nicht den ersten Schritt, doch dieser war nicht einmal bereit, sich in die Hauptstadt zu begeben. So war Anna es, die ihren Sohn dazu bewegen sollte, nach Paris zu kommen und über einen Frieden zu verhandeln. Die Königinmutter, schreibt Lucinge, a pressé Mme de Nemours de se haster plustost à cet effect que pour les nopces ${ }^{100}$.

Bald hatten alle verstanden, daß der König diese Heirat nicht unterstützte, daß er seine Nichte lieber Heinrich von Navarra gab und erst die Geburt eines möglichen Savoyer Erbprinzen abwarten wollte. Allein Anna tat sich schwer,

${ }^{98}$ Anna d'Este an Catalina Micaela de Austria, 26. Dez. (1585), Annecy, in: AST (Cast.), Int., Let. div., Prin. div. 78. Die Einigung über das douaire, 15. Dez. 1585, in: AST (Cast.), Int., PGN, cat. 2, m. 13, n. 14. Vgl. Gherardo Bonmercati an Alfonso d'Este, 10. Dez. 1585, Turin, in: ASM, Canc. duc., Amb. Francia 96; Katharina von Medici an Anna d'Este, 15. Dez. 1585, Paris, in: MÉDICIS, Lettres, Bd. 8, S. $372 \mathrm{f}$.

${ }_{99}$ Girolamo Ragazzoni an Girolamo Rusticucci, 28. Apr. 1586, Paris, in: Correspondance de la Nonciature de Girolamo Ragazzoni, évêque de Bergame, nonce en France 1583-1586, hg. v. Pierre BLET, Rom, Paris 1962, S. 569; Edward Stafford an Francis Walsingham, 24. Jan. 1586, 1. Okt. 1587, Paris, in: Calendar Elizabeth, 1921, S. 314, 1927, S. 384. Das Zitat: René de Lucinge an Carlo Emanuele di Savoia, 20. Jan. 1586, Paris, in: LuCINGE, Lettres 1586, S. 37.

${ }^{100}$ René de Lucinge an Carlo Emanuele di Savoia, 12. Febr. 1586, Paris, in: LuCINGE, Lettres 1586, S. 66f. Vgl. Edward Stafford an Francis Walsingham, 4. April u. 15. Juli 1586, Paris, in: Calendar Elizabeth, 1921, S. 518, 1927, S. 52. 
die Tatsachen zu akzeptieren. Le Roy se servira d'elle et luy donnera belles parolles tandis qu'il voudra fonder asseurance entre lui et Mr. de Guise: car un chascun pense et croyt qu'il n'afectionne aucunement ce mariage, beobachtete der Botschafter Savoyens und stellte fest, daß die Sache auf einen Tauschhandel herauslaufen würde: Die Königinmutter habe ihre Freundin zum vray instrument de la paix auserkoren ${ }^{101}$. Dies zumindest hatte die Prinzessin verstanden, und so versuchte sie, ihren ältesten Sohn zu Friedensverhandlungen zu bewegen. Doch Henri blieb hart. Ende März 1586 sah Anna ihre letzten Hoffnungen schwinden, bei Hofe erschien sie plus mélancolique que de coustume. Tatsächlich wurde sie krank und mußte das Bett hüten, più che molto travagliata delle lunghezze che le loro Maestà danno a questo maritaggio; den Bruder bat sie in dieser Zeit, avoyr pytié de mes annuyz ${ }^{102}$. Mit dem Erstarken der Ligue wurde die Heirat des Herzogs von Nemours zu einem Politikum. Anna hatte nun überhaupt keinen Einfluß mehr auf das Geschehen, sie konnte den Schwankungen des königlichen Willens nur noch sprachlos folgen, und oft genug wußte sie nicht, à quel sainct se rendre ${ }^{103}$.

Mitte April 1587 war die Prinzessin einem Nervenzusammenbruch nahe, einen ganzen Tag lang weinte sie à grosses larmes und war auch von der Königinmutter nicht zu trösten. Nicht ohne Ironie bemerkte Lucinge, die Herzogin, qui est spirituelle, habe nun wohl doch verstanden, daß der König diese Heirat nicht wolle, und ein anderer Beobachter stellte fest: Di qui nascono le soavi lagrime di madama di Nemours, la quale [...] piange di continuo nel grembo del Re e della Reina Madre ${ }^{104}$. Während bereits Gerüchte uber eine Verbindung der Lothringer Prinzessin mit dem Sohn des Herzogs von Parma oder dem Großherzog von Toskana kursierten, versuchte Anna, die den Hof voller Wut verlassen und sich in ihr Hôtel de Nemours zurückgezogen hatte, zumindest nach außen hin die Illusion einer bevorstehenden Hochzeit aufrechtzuerhalten, selbst als ihre Söhne nach dem Tag der Barrikaden jeglichen verbliebenen Rest von königlichem Wohlwollen verloren hatten ${ }^{105}$. Im Sep-

${ }^{101}$ René de Lucinge an Carlo Emanuele di Savoia, 2. Febr. u. 25. Mărz 1586, Paris, in: LUCINGE, Lettres 1586, S. 50, 129.

102 René de Lucinge an Carlo Emanuele di Savoia, 25. März 1586, Paris, in: LuCINGE, Lettres 1586, S. 129; Gherardo Bonmercati und Anna d'Este an Alfonso d'Este, 30. Mărz 1586, Paris, in: ASM, Canc. duc., Amb. Francia 96 u. Cart. prin. est. 1458/22.

${ }^{103}$ René de Lucinge an Carlo Emanuele di Savoia, 3. Mai 1587, Paris, in: LuCINGE, Lettres 1587 , S. 169.

${ }^{104}$ René de Lucinge an Carlo Emanuele di Savoia, 15. Apr. u. 8. Juli 1587, Paris, in: LuCINGE, Lettres 1587, S. 128, 234; Filippo Cavriana an Belisario Vinta, 12. Aug. 1587, in: DESJARDINS (Hg.), Négotiations diplomatiques, Bd. 4, S. 707.

${ }^{105}$ Anna d'Este an Carlo Emanuele di Savoia, 27. Okt. 1587, Paris, in: AST (Cast.), Int., Let. div., Prin. div. 78; Dies. an Alfonso d'Este, 9. Aug. 1588, Paris, in: ASM, Canc. duc., Cart. prin. est. 1458/22; Carlo Emanuele di Savoia an den Chevalier d'Elbene, 6. März 1588, Turin, in: MÉDICIS, Lettres, Bd. 8, S. 486f.; Edward Stafford an Francis Walsingham, 12. Apr. 1588, Paris, in: Calendar Elizabeth, 1927, S. 591. 
tember 1588 war das Eheprojekt endgültig gescheitert. Zu ersehen ist dies aus einer Zahlungsanweisung des Königs an die Herzogin von Nemours, die dieser wohl weniger freiwillig als vielmehr durch die Macht der Guise gezwungen unterzeichnet hatte. In besagtem Papier weist Heinrich seine Schatzmeister an, der Prinzessin die Summe von 100000 Écus auszuzahlen, da er sie für die im Rahmen der geplanten Heirat ihres Sohnes aufgebrachten grands frais et despenses entschädigen wolle. Nur Charles-Emmanuel scheint sich mit der erlittenen Niederlage nicht abgefunden zu haben und drohte, seine einstige Braut auf ihrem Weg nach Florenz zu entfuhren ${ }^{106}$.

\section{Bemühungen um die Karrieren der Söhne}

Einige weitere Beispiele machen deutlich, in welchem Maße Erfolg oder Mißerfolg der Vorhaben Annas von den Entwicklungen der allgemeinen politischen Situation abhängig waren: die Emennung von Charles-Emmanuel zum Gouverneur von Lyon und die Versorgung ihres jüngsten Sohnes Henri mit einem Teil der Pfrunde seines Onkels. Noch zu Lebzeiten des Vaters war der Prinz von Genevois als Lyoner Gouverneur vorgesehen, doch später versprach Heinrich den Posten einem seiner mignons, wogegen die Herzogin von $\mathrm{Ne}-$ mours lautstark protestierte ${ }^{107}$. In den folgenden Jahren genoß Anna bei ihrem Vorhaben die Unterstützung der Königinmutter, vielleicht, weil diese das Heiratsprojekt fur den jungen Herzog von Nemours als aussichtslos betrachtete und der Freundin wenigstens einen ihrer Wünsche erfullen wollte. Das Gerücht vom Tod des bisherigen Gouverneurs kursierte nun öfter bei Hofe, manchmal wurde vermutet, Anna selbst hätte es in die Welt gesetzt, um den königlichen Willen ihrem Sohn gegenüber zu prüfen ${ }^{108}$. Es bestätigte sich schließlich Ende November 1588, als der König unter enormen Druck seitens der Ligue stand und gar nicht anders konnte, als den Posten dem Herzog von Nemours zu uberlassen, offiziell zwar à l'instante prière et requête de ma-

${ }^{106}$ Heinrich III. an seine Schatzmeister, 30. Sept. 1588, Blois, Kopie, in: BnF, Fr. 18709, fol. 592r. Die Auszahlung dieses Geldes wurde von Heinrich IV. im Vertrag von Folembray vom 11. Jan. 1596 bestătigt: Ibid. fol. 588v. Eine Quittung über diese Summe ist verzeichnet in: Extrait des pieces produites par Monsieur le Duc de Nemours aux fins d'estre maintenu en la possession et jouissance du comté de Gisors, ainsi qu'il a esté delaissé à feủe Madame Renée de France son ayeule, o.D. (17. Jh.), in: BnF, Fr. 17308, fol. 41-47, hier fol. 44v. Orazio Rucellai an Pietro Usimbardi, 12. Febr. 1589, in: DESJARDINS (Hg.), Négotiations diplomatiques, Bd. 4, S. 880.

${ }^{107}$ Edward Stafford an Francis Walsingham, 2. Apr. 1584, Paris, in: Calendar Elizabeth, 1914 , S. 445.

${ }^{108}$ Edward Stafford an Francis Walsingham, 7. u. 24. Juni 1586, Paris, in: Calendar Elizabeth, 1927, S. 7f., 21. 
dame de Nemours, doch trotzdem gezwungenermaßen, konnte er doch die Wünsche der Guise und ihrer Verblindeten nur schlecht ignorieren ${ }^{109}$.

Parallel zu den Heiratsverhandlungen für Charles-Emmanuel bemühte sich Anna um die Karriere ihres jüngsten Sohnes, der Kardinal werden sollte. Sie bat den König, noch zu Lebzeiten ihres Bruders einen Teil von dessen Pfründen in Frankreich an Henri zu übertragen, gleichzeitig sollte der Knabe bei Luigi in die Lehre gehen, um auf die kirchliche Laufbahn vorbereitet zu werden. So schnell wie möglich, das hieß à la premyère promotyon qu'an fera sa Sayntété, wünschte die Prinzessin, den Marquis in Purpur gewandet zu sehen $^{110}$. Doch Annas Plăne wurden von der Reform des Kardinalskollegiums durch den Papst durchkreuzt. Zwei Brüder, oder ein Onkel und sein Neffe, könnten zukünftig nicht mehr zur gleichen Zeit Kardinal sein, und Henri habe schon einen Bruder und einen Onkel an der Kurie, erläuterte Luigi der Schwester die neuen Bestimmungen und tröstete, daß Henri zu Lebzeiten dieses Papstes zwar nicht Kardinal werden könne, mais il viendra un autre Pape qui fera a sa façon comme cestuici faict a la sienne. Nach Luigis Tod bat Anna den König, ihren Sohn nicht zu vergessen, da er le plus jeune et le plus paure de tous sei $^{111}$. Wie schon bei der Ernennung von Charles-Emmanuel zum Gouverneur von Lyon wird auch diesmal die Überlegung Katharinas eine Rolle gespielt haben, der Freundin angesichts des vorauszusehenden Scheiterns der Heirat des Herzogs von Nemours wenigstens in einem kleineren Wunsch entgegenzukommen. Heinrich willigte zwar ein und überließ dem Marquis das Erzbistum Auch sowie zwei Abteien, doch handelte es sich hierbei um den weniger attraktiven Teil, während nicht wenige von Luigis besseren Pfrunden an den Kardinal von Joyeuse gingen. Wahrscheinlich als Ausgleich bemühte Katharina sich dann beim Papst um die kostenlose Ausfertigung der notwendigen Papiere ${ }^{112}$.

${ }^{109}$ L'ESTOILE, Journal Henri III, S. 577. Vgl. das Glückwunschschreiben der Stadt Lyon an Anna d'Este u.a. diesbezulgliche Dokumente, Anfang Dez. 1588, in: Jean-Hippolyte MARIÉJOL, Charles-Emmanuel de Savoie, duc de Nemours, gouverneur du Lyonnais, Beaujolais et Forez (1567-1595), Paris 1938, S. 67. Einen Monat später bestătigte Heinrich III. die Ernennung, vgl. sein Memorandum für Giovanni Francesco Morosini, Nuntius in Frankreich, ca. Febr.-März 1589, in: Richard COOPER, The Aftermath of the Blois Assassinations of 1588: Documents in the Vatican, in: French History 3 (1989) S. 404-426, hier S. 418.

${ }^{110}$ Anna d'Este an Luigi d'Este, o.D. (Okt. 1586), Paris, in: ASM, Canc. duc., Cart. prin. est. $1459 / 23$.

${ }^{111}$ Luigi d'Este an Anna d'Este, 15. Dez. 1586, Rom, in: MÜNCH (Hg.), Denkwürdigkeiten, S. 258; Anna d'Este an Alfonso d'Este, 24. Jan. 1587, Paris, in: ASM, Canc. duc., Cart. prin. est. 1458/22. Tatsăchlich sollten die Reformen Sixtus' V. bis ins 20. Jh. Bestand haben.

${ }^{112}$ René de Lucinge an Carlo Emanuele di Savoia, 8. Febr. 1587, Paris, in: LUCINGE, Lettres 1587, S. 42; Katharina von Medici an den Marquis de Pisany, französischer Gesandter in Rom, und an Alessandro Peretti, Kardinal von Montalto, 15. Febr. u. 20. Sept. 1587, in: MÉDICIS, Lettres, Bd. 9, S. 171, 236f. 
Zehn Jahre später war von einer kirchlichen Laufbahn für Annas jüngsten Sohn nicht mehr die Rede. Henri hatte sein fünfundzwanzigstes Lebensjahr erreicht und war volljährig, seit dem Tod des Bruders im Sommer 1595 trug er den Herzogstitel. Anstatt den Kardinalshut zu erlangen galt es nun, eine Heirat für ihn zu verhandeln, doch die Gespräche verliefen kaum erfolgreicher als die für das Eheprojekt von Charles-Emmanuel. Wann und wie er dazu gekommen war, eine Verbindung mit Catherine d'Orléans, Tochter eben jener Herzoginwitwe von Longueville ins Auge zu fassen, gegen die Henris Mutter seit Jahren einen noch nicht endgültig entschiedenen Prozeß fuhrte, ist unklar. Im Dezember 1598 jedenfalls beschloß der König, sich in die festgefahrenen Verhandlungen einzuschalten, und er schrieb sowohl an Anna als auch an die Mutter der Braut: je veux estre juge de ce differend. Da es derzeit äußerst schwierig sei, das zu finden, was man wünsche, sollten sie nicht starr an ihren Entscheidungen festzuhalten, de peur que si ceste occasion vous eschappe, vous ne la regrettiés ${ }^{113}$. Die Damen scheinen dem königlichen Befehl Folge geleistet zu haben, denn schon wenige Monate später war der Vertrag aufgesetzt, und Anna bat den Herzog von Savoyen um seine Zustimmung. Carlo Emanuele leitete trèsvoulentiers et promptement die nötigen Schritte ein und sprach dem Bräutigam seine Glückwünsche aus ${ }^{114}$. Doch während im Sommer 1599 noch von der Hochzeit des Herzogs von Nemours die Rede war, schejnen sich in der Folge größere Probleme ergeben zu haben, denn der Ehevertrag wurde nie unterzeichnet, und Carlo Emanuele mußte sich bald erneut über den Stand der Dinge informieren. Daß das Projekt kurz darauf endgültig scheiterte, ist an der Vollmacht abzulesen, mit welcher der Herzog von Nemours seine Mutter zur Weiterführung des Prozesses gegen die Longueville ermächtigte $^{115}$.

Die gescheiterten Bemühungen der Herzogin von Nemours um Eheverbindungen und Karrieren der Söhne zeigen, in welchem Maße das Gelingen von Annas Plänen von der aktuellen Situation abhängig war, und daß in manchen

${ }^{113}$ Heinrich IV. an Anna d'Este, 11. Dez. 1598, in: HENRI IV, Recueil de lettres missives, Bd. 5, hg. v. Jules BERGER DE XIVREY, Paris 1850, S. 73f. Vgl. die Briefe an Marie de Bourbon, Françoise d'Orléans, Diane de France, Henriette de Clèves, 11. Dez. 1598, in: Ibid. S. 74-77.

114 Anna d'Este an Carlo Emanuele di Savoia, 2. Apr. (1599), Paris, in: AST (Cast.), Int., Let. div., Prin. div. 78; Carlo Emanuele di Savoia an Anna d'Este und Henri de Savoie, 13. Mai 1599, Turin, in: BnF, Fr. 3418, fol. 77r, 79r; Erlaubnis von Carlo Emanuele di Savoia für Henri de Savoie, seiner Braut Pensionen und douaire zuzuweisen, 13. Mai 1599, in: Ibid. PGN, cat. 2, m. 14, n. 4; ein Entwurf des Heiratsvertrags in: BnF, Fr. 2991, fol. 75f.

115 Vollmacht von Henri de Savoie für Anna d'Este, 26. Aug. 1600, in: AST (Cast.), Int., PGN, cat. 4, m. 4, n. 13; Carlo Emanuele di Savoia an Henri de Savoie, 6. Okt. 1599, Kopie, in: Ibid. Let. div., Du. sov. 25; Gasparo Silingardi an Pietro Aldobrandini, 18. Juli 1599, Paris, in: Correspondance du nonce en France Gasparo Silingardi, évêque de Modène (15991601), hg. v. Bertrand HAAN, Rom 2002, S. 299. Erst 1618 sollte sich Henri de Savoie mit Anne de Lorraine vermählen. 
Fällen selbst ihr gutes Verhältnis zu Katharina von Medici nicht weiterhalf. Mit der fortschreitenden Emanzipierung Heinrichs von seiner Mutter schrumpften deren Möglichkeiten zur Einflußnahme zugunsten der Freundin, so daß die Prinzessin nun in größerem Maße auf die Gunst des Königs selbst angewiesen war. Gehörten ihre Söhne, vor allem die aus erster Ehe, dank der Entwicklungen der Zeit zu den Mächtigen Frankreichs, wurde Annas Wünschen eher entsprochen, ansonsten konnte sie oftmals nur hoffen und warten.

\section{Anna und die Ligue}

Die Frage, welche Position die Herzogin von Nemours während der Ereignisse der 1580er Jahre bezog und in welchem Verhältnis sie zur Ligue stand, kann nicht eindeutig beantwortet werden. In den Quellen erscheint sie meist als diejenige, welche die Söhne zu Vernunft und Mäßigung ermahnte. So schildert etwa Philippe Duplessis-Mornay in einer seiner an die Reformierten gerichteten Schriften, wie der Herzog von Guise an einem Nachmittag im März 1584 von seiner Mutter stundenlang ermahnt worden war, de plier à tout [...], et ne se formaliser de rien, nommément de s'abstenir (c'étoient ses mots) de faire des boutades contres les Mignons. Und Pierre de L'Estoile, der ansonsten nicht immer nur gute Worte fur die Prinzessin übrig hat, behauptet, Jacques de Savoie habe auf dem Sterbebett die Ligue verwünscht und mit Blick auf seine Gemahlin prophezeit, qu'elle leur gâterait tout. Bei beiden Erzählungen handelt es sich um erfundene Anekdoten, doch zeigt ihre Erwähnung durch Autoren, die selbst nicht dem Lager der radikalen Katholiken angehörten, daß auf die Herzogin nicht automatisch die Meinung ihrer Söhne übertragen wurde. Brantôme hingegen erwähnt das Interesse der Prinzessin an der Vereinigung zumindest in deren Anfangszeit ${ }^{116}$. In den Schilderungen der ausländischen Gesandten schließlich erscheint Anna teils als Gegnerin oder Kritikerin der Ligue, teils als deren Parteigängerin. Die Herzogin von Nemours sei mit ihren Söhnen nicht sehr zufrieden, war etwa im März 1587 in Florenz zu erfahren, perciocchè non approva la Lega condotta come la si conduce. Vom selben Informanten war die Prinzessin ein Jahr zuvor als diejenige beschrieben worden, la quale ha ravvivato i partigiani, die also die Stimmung in Paris ange-

\footnotetext{
${ }^{116}$ Philippe DUPLESSIS-MORNAY, Lettre de Discours, sur les divers jugemens des occurrences du temps du 15. [nicht 18.!] Mars 1584, o.O. o.J. [1584], in: Mémoires de la Ligue, contenant les évenemens les plus remarquables depuis 1576, jusqu'à la Paix accordée entre le Roi de France et le Roi d'Espagne, en 1598, Nouvelle édition, Bd. 1, Amsterdam 1758, S. 584-595, Zitat S. 589f.; L'EsTOILE, Henri III, S. 383f.; BRANTÔME, Recueil des Dames, S. 709f. Vgl. den Bericht von Guillaume Stuart de Vézines, 23. Jan. 1583, Paris, in: BézE, Correspondance, Bd. 24, S. 343, aus dem hervorgeht, daß Anna die katholischen Schweizer Kantone bei ihrem Vorgehen gegen Genf unterstützte.
} 
heizt habe und so ihrem Sohn einen triumphalen Einzug in die Stadt verschaffen konnte, atteso che ella è molto ben vista in questa cittá ${ }^{117}$.

Katharina von Medici wußte um den Einfluß der Herzogin auf ihre Söhne. Einige Jahre zuvor hatten sie und der König bereits davon profitiert, indem sie die Guise durch ihre Mutter an den Hof hatten zurlickrufen lassen, doch nun war Heinrich skeptisch hinsichtlich Annas mütterlicher Autorität. Der Savoyer Gesandte berichtet von einem Streitgespräch zwischen Katharina und ihrem Sohn, bei dem die Italienerin die Meinung vertrat, der Herzog von Guise könne von seiner Mutter zur Annahme eines Friedens überredet werden. Heinrich antwortete, daß Kinder bei diesen Fragen nie auf ihre Mütter hörten, worauf Katharina erwiderte, Annas Söhne hätten sich immer gehorsam gezeigt und den Worten der Herzogin stets Glauben geschenkt. Der König jedoch, so der Bericht, ne voulut jamais accorder à sa mère que Madame de Nemours fusse propre à y estre emploiée, ou qu'on luy doyve sçavoir gré pour cela ${ }^{118}$. Zwar war ihr Sohn anderer Meinung, doch wußte Katharina sich ihrer Freundin zu bedienen. Die Königinmutter, ließ Anna ihren Bruder wissen, als in Epernay uber den späteren Vertrag von Nemours verhandelt werden sollte, $m$ 'a prié de mander et commander à mesdictz enfans de ne s'y rendre difficiles, ains de s'y accommoder, und Lucinge erwăhnt, die Prinzessin habe auf Katharinas Bitte hin den Sohn aufgefordert, einige seiner demandes exorbitantes fallen zu lassen. Im Mai 1587 ließ sich Katharina dann, um über eine bessere Verhandlungsbasis zu verfugen, von ihrer Freundin und anderen Fürstinnen und Fürsten zu einem Treffen mit Vertretern der Ligue in Reims begleiten, und im folgenden Jahr trafen sich die beiden Damen bei der Zusammenkunft zwischen dem König und dem Herzog von Guise in Chartres wieder ${ }^{119}$.

$\mathrm{Da}$ für die Prinzessin jedoch die Sicherheit und das Wohl ihrer Sobhne nicht weniger wichtig waren als eine vernünttige Politik oder ein gutes Verhältnis zum König, zeigen die Ereignisse des Jahres 1587. Als der Herzog von Mayenne aus Angst vor Giftattentaten nach Paris zu kommen zögerte, beruhigte ihn die Mutter zwar zunächst, doch als er sich dann in der Stadt befand, ermahnte sie ihn zur Vorsicht, avec des frayeurs telles que les mères ont

${ }^{117}$ Filippo Cavriana an Belisario Vinta, 3. März 1587, 3. März 1586, in: DESJARDINS (Hg.), Négotiations diplomatiques, Bd. 4, S. 680, 635 .

${ }^{118}$ René de Lucinge an Carlo Emanuele di Savoia, 4. Febr. 1586, Paris, in: LuCINGE, Lettres 1586, S. 52f. Zu den früheren Ereignissen: Sinolfo Saracini an Francesco de' Medici, 9. Febr. 1579, in: DESJARDINS (Hg.), Négotiations diplomatiques, Bd. 4, S. 242f.; Anselmo Dandino an Tolomeo Galli, 18. Febr. 1579, Paris, in: Correspondance Dandino, S. 328.; Katharina von Medici an Anna d'Este, 8. März 1579, Agen, in: MÉdICIS, Lettres, Bd. 6, S. 296.

${ }^{119}$ Anna d'Este an Alfonso d'Este, o.D. (April 1585), in: ASM, Canc. duc., Cart. prin. est. 1458/22; René de Lucinge an Carlo Emanuele di Savoia, 23. Mai 1585, Paris, in: LuCINGE, Lettres 1585, S. 94. Die Mitteilung über den Abschluß des Friedens: Charles de Bourbon an Anna d'Este, 8. Juli (1585), Nemours, in: BnF, Fr. 3367, fol. 29r. Vgl. Le PERSON, Practiques, S. $183 \mathrm{f}$., 538. 
accoustumé pour la conservation de leur portée ${ }^{120}$. Nachdem die deutschen Reiter, in Frankreich als wreitres" bekannt und berlichtigt, im Sommer den Rhein uberschritten hatten und das Königreich in Schutt und Asche zu legen drohten, begaben sich auch die Herzöge von Guise und von Nemours auf das Schlachtfeld. Anna hatte diese Entscheidung zunächst nicht akzeptieren wollen und die Söhne mit aller Macht zurückzuhalten versucht, doch dann bemühte sie sich um praktischen Beistand. Im Oktober bat sie den Herzog von Savoyen, Charles-Emmanuel eine Truppe leichter Reiter et quelque sage capitaine bereitzustellen, pour assister près de sa personne durant lesdits troubles. Henri de Lorraine schlug die reîtres dann vor Vimory und Auneau, und es war ganz natürlich, daß die Glückwünsche an die Mutter des Helden gingen noch bevor sie dem Konig ausgerichtet wurden ${ }^{121}$.

Die Freundschaft der Prinzessin zur Königinmutter war all diese schwierigen Jahre lang ungetrubt, sieht man von den voribergehenden Spannungen wegen des unerfullten Heiratsversprechens für Charles-Emmanuel ab. $L a$ royne continue tousiours à m'escrire et me monstrer l'affection acoustumée qu'elle me porte, schrieb Anna zu einem Zeitpunkt, als ihre Söhne kurz davor waren, dem König den Text des Vertrags von Nemours zu diktieren, und ein knappes Jahr später sah man Katharina die melancholische Freundin auf rührende Weise trösten ${ }^{122}$. Auch zur regierenden Königin und den meisten Mitgliedern des Hofes unterhielt die Herzogin ein gutes Verhältnis. Als sie etwa nach langer Abwesenheit und dem Tod ihres Gemahls Ende Januar 1586 in Paris eintraf, wurde Anna von fast dem gesamten Hof außerhalb der Stadtmauern erwartet und zu den Tuilerien geleitet, wo Königin Luise sie avec fort grande démonstration d'amitié empfing ${ }^{123}$.

${ }^{120}$ René de Lucinge an Carlo Emanuele di Savoia, 24. Jan. u. 3. Mărz 1587, in: LuCINGE, Lettres 1587, S. 30, 74.

121 Anna d'Este an Carlo Emanuele di Savoia, 27. Okt. 1587, Paris, in: AST (Cast.), Int., Let. div., Prin. div. 78. Anna nahm an dem Te Deum teil, das Heinrich III. am 23. Dez. 1587 zur Feier dieses Sieges singen ließ: Le Retour du Roy à Paris après la desfaicte de l'armée des estangers, o.D. (23. Dez. 1587), Kopie des 17. Jh., in: BnF, Fr. 4337, fol. 80v; L'EstollE, Henri III, S. 507. Zu ihrer Anwesenheit bei einem weiteren Te Deum, 19. Juli 1588: Xavier LE PERSON (Hg.), Journal d'un ligueur parisien. Des barricades à la levée du siège de Paris par Henri IV (1588-1590), Genf 1999, S. 101f.

${ }^{122}$ Anna d'Este an Alfonso d'Este, o.D. (April 1585), in: ASM, Canc. duc., Cart. prin. est. 1458/22; Edward Stafford an Francis Walsingham, 14. Mărz 1586, Paris, in: Calendar Elizabeth, 1921 , S. 442 .

${ }^{123}$ René de Lucinge an Carlo Emanuele di Savoia, 1. Febr. 1586, Paris, in: LUCINGE, Lettres 1586, S. 18. 


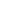

